\title{
Atomistic modeling of nanoparticle generation in short pulse laser ablation of thin metal films in water
}

\author{
Cheng-Yu Shih, Chengping Wu, Maxim V. Shugaev, and Leonid V. Zhigilei \\ Department of Materials Science and Engineering, University of Virginia, \\ 395 McCormick Road, Charlottesville, Virginia 22904-4745, USA
}

\begin{abstract}
Laser ablation in liquids is actively used for generation of clean colloidal nanoparticles with unique shapes and functionalities. The fundamental mechanisms of the laser ablation in liquids and the key processes that control the nanoparticle structure, composition, and size distribution, however, are not yet fully understood. In this paper, we report the results of first atomistic simulations of laser ablation of metal targets in liquid environment. A model combining a coarse-grained representation of the liquid environment (parameterized for water), a fully atomistic description of laser interactions with metal targets, and acoustic impedance matching boundary conditions is developed and applied for simulation of laser ablation of a thin silver film deposited on a silica substrate. The simulations, performed at two laser fluences in the regime of phase explosion, predict a rapid deceleration of the ejected ablation plume and the formation of a dense superheated molten layer at the water-plume interface. The water in contact with the hot metal layer is brought to the supercritical state and transforms into an expanding low density metal-water mixing region that serves as a precursor for the formation of a cavitation bubble. Two distinct mechanisms of the nanoparticle formation are predicted in the simulations: (1) the nucleation and growth of small (mostly $\leq 10 \mathrm{~nm}$ ) nanoparticles in the metal-water mixing region and (2) the formation of larger (tens of nm) nanoparticles through the breakup of the superheated molten metal layer triggered by the emergence of complex morphological features attributed to the Rayleigh-Taylor instability of the interface between at the superheated metal layer and the supercritical water. The first mechanism is facilitated by the rapid cooling of the growing nanoparticles in the supercritical water environment, resulting in solidification of the nanoparticles located in the upper part of the mixing region on the timescale of nanoseconds. The computational prediction of the two mechanisms of nanoparticle formation yielding nanoparticles with different characteristic sizes is consistent with experimental observations of two distinct nanoparticle populations appearing at different stages of the ablation process.
\end{abstract}




\section{Introduction}

Following the initial report of the nanoparticle synthesis by pulsed laser ablation in liquids (PLAL) [1] and subsequent demonstrations of the versatility of this method in generation of noble metal nanoparticles [2-5], the investigations of laser ablation in liquids have evolved into an active research area. The steadily growing interest in PLAL [6] is largely defined by the good productivity and process-stability of this technique [7,8], combined with its ability to produce stable contamination-free colloidal solutions of nanoparticles suitable for applications in various fields, including biomedicine [9,10], chemical catalysis [11-14], and plasmonics [15,16].

The characteristics of nanoparticles synthesized by PLAL can be affected by the choice of the liquid medium [17-19], its temperature [20], as well as the introduction of organic ligands [21-24] or inorganic salts [25] into the liquid environment. Post irradiation of colloidal solutions of nanoparticles produced by PLAL has been demonstrated to be an effective way to further modify the size, shape and composition of the nanoparticles through laser induced melting, evaporation, and fragmentation in liquids [26-30]. Moreover, the highly nonequilibrium conditions created by the interaction of the ablation plume with liquid environment can result in the formation of nanoparticles with unusual structure, shape and composition, such as nanocubes [31], hollow spheroids [32,33], patch-joint football-like AgGe microspheres [34], and diamond nanocrystallites produced by laser ablation of graphite [35].

Further optimization of experimental parameters in PLAL for the efficient generation of nanoparticles with structures, compositions and size distributions fine-tuned to the needs of practical applications can be facilitated by improved physical understanding of the involved processes. Despite the continuous advancements in the application of PLAL for production of nanoparticles for an increasingly wide range of material systems [36-40] and targeted experimental studies aimed at reveling the key processes responsible for the nanoparticle generation [41-47], the fundamental mechanisms of the nanoparticle formation by laser ablation in liquids are still not fully understood.

The general picture of laser ablation in liquids is very different from the one in vacuum. The ablation plume does not expand freely but is confined by the liquid environment. The liquid in contact with the plume is quickly heated and vaporized to form a thin layer of vapor surrounding the plume. This thin vapor layer can be directly observed via shadowgraphy [42] or X-ray 
radiography [45] as a dark zone surrounding the plume. The supply of heat from the plume creates and supports high temperature and pressure conditions in the vapor layer, and drives the expansion of the layer. The expansion of the vapor layer away from the target leads to the formation of a cavitation bubble, while the pressure it exerts in the opposite direction pushes against the ablation plume and suppresses its expansion.

Currently, an accepted view is that the ablated material is likely to be confined and trapped inside the cavitation bubble, where favorable pressure and temperature conditions for nanoparticle nucleation, growth, coalescence, and solidification are realized $[41,48]$. This general scenario, however, cannot be directly verified and detailed with conventional optical methods, since an optically dense interface between the liquid and the cavitation bubble blocks the view of the processes occurring inside the bubble. Recently, the novel use of small angle Xray scattering (SAXS) is demonstrated to be capable of relating the cavitation bubble dynamics to nanoparticle growth [43-45]. In-situ SAXS can be used to scan different positions in the bubble with different time delays, thus enabling mapping of the nanoparticle size distribution with respect to the time and position inside the bubble [43-45].

Signals yielded by SAXS have revealed two distinct nanoparticle size populations in the cavitation bubble, the "primary particles," with size distribution centered around 8-10 nm, and "secondary particles," with sizes around $50 \mathrm{~nm}$. The primary particles are detected at the early stage of the first bubble expansion and are likely to be directly ejected from the irradiated target or formed during the first nanosecond of the plume expansion, which is currently beyond the temporal resolution of SAXS. Higher density of primary particles is detected near the bottom of the bubble, and their abundance decays toward the top of the bubble. Some of the primary particles are detected in the liquid outside the cavitation bubble, suggesting that the initial fast plume expansion causes injection of vaporized atoms into the liquid followed by the formation of nanoparticles at a later stage. The signal from the secondary particles is weak during the first cycle of the bubble expansion and collapse, but becomes stronger after the rebound. The secondary particles are speculated to form due to the agglomeration of primary particles during the bubble collapse, when the primary particles are forced to collide with each other due to the sudden volume contraction. The sizes of the secondary particles are found to be highly variable, with rapid changes observed in the course of the bubble dynamics, suggesting that these 
"particles" may not be compact objects, but some forms of loose networks of molten material or nanoparticle agglomerates.

While the SAXS data provides first hand quantitative insights into the nanoparticle generation in PLAL, other approaches are needed to fully capture the nanoparticle generation mechanisms. In particular, the atomic clusters and nanoparticles smaller than $5 \mathrm{~nm}$ cannot be detected with SAXS but may be responsible for the nanoparticle nucleation and growth in the ablation process as well as the slower growth and coarsening of nanoparticles in the colloidal solutions generated by PLAL [49,50]. One needs to push beyond the current SAXS limits on the particle sizes and temporal/spatial resolution to observe the ablation phenomena in more details. Moreover, there is a need to resolve the thermodynamic states of the plume confined in the cavitation bubble to enable reliable theoretical description of PLAL.

The complexity and highly nonequilibrium nature of processes involved in PLAL has been preventing a rigorous theoretical treatment of nanoparticle formation in liquids As a result, the theoretical analysis of the nanoparticle formation by laser ablation in liquids [51] has been largely based on semi-quantitative models that adopt the concepts developed for the plume expansion in a background gas to the much stronger confinement by a liquid environment, and describe the nanoparticle formation as a process of coalescence of clusters in a supersaturated solution formed by the mixing of the ablation plume and the liquid. The analysis in this case relies on the assumptions of the initial cluster size distribution in the solution, the temperature evolution in the plume-liquid mixing region, the thickness of the mixing region, and other parameters.

The difficulties of the theoretical analysis are shared by continuum-level computational approaches, where realistic modeling of phase transformations occurring under highly nonequilibrium conditions represents a daunting challenge. The first hydrodynamic simulations of PLAL [52,53] adopt a simplified representation of water environment as a thermally insulating, non-mixable overlayer described by a single-phase equation of state. The highly simplified representation of the water environment prevents the realistic description of some of the key processes, such as the formation of a layer of water vapor driving the expansion of the cavitation bubble, water-metal mixing and rapid cooling of the metal species in the mixing region. Moreover, the 1D nature of the model does not allow for the direct simulation of the 
nanoparticle generation. Nevertheless, the results of the first hydrodynamic simulations have provided important insights into the evolution of thermodynamic conditions and phase decomposition at the early stage of PLAL.

Under conditions when theoretical description and continuum-level modeling of PLAL are hindered by the complexity and highly nonequilibrium nature of laser-induced processes, the molecular dynamics (MD) computer simulation technique $[54,55]$ can serve as a useful alternative approach, capable of providing atomic-level insights into the laser-induced processes. The main advantage of the MD technique is that no assumptions are made on the processes or mechanisms under study. The only input in the MD model is the interatomic interaction potential that fully defines the structure and thermodynamic properties of the material. The MD technique has indeed been successfully applied to simulations of laser melting, spallation and ablation of molecular systems, metals, and semiconductors in vacuum, as reviewed in Refs. [5659]. There have been no MD simulations of PLAL, however, attempted so far. The high computational cost of the atomistic representation of both the target material and liquid environment combined with the relatively large time- and length-scales of processes responsible for the nanoparticle generation in PLAL have been discouraging the application of the MD technique to simulation of laser-materials interactions in liquid environment.

In this paper, we report the results of the first MD simulations aimed at revealing the nanoparticle formation mechanisms in PLAL. The target in the simulations of laser ablation is a thin Ag film deposited on a transparent silica substrate. The use of the thin film rather than a bulk metal target commonly used for nanoparticle production in PLAL is allowing us to perform the first exploration of the effects of the ablation plume confinement by liquid environment and nanoparticle formation mechanisms at a reduced computational cost. Moreover, the exploration of the role of the spatial confinement of the deposited laser energy within a thin metal film may provide ideas for achieving a more precise control over the size distribution of nanoparticles generated in PLAL [60,61]. The simulations are enabled by the development of a computationally-efficient coarse-grained representation of liquid environment combined with the acoustic impedance matching boundary conditions that allow us to focus the computational efforts on the part of the system where the active processes of laser ablation, metal-liquid mixing, and nanoparticle formation take place. The computational model and its parametrization for simulation of laser ablation of a thin Ag film in water environment are described below, in 
Section 2. The results of large-scale MD simulations performed at two laser fluences and the implications of the computational predictions for interpretation of the experimental data on the nanoparticle formation in PLAL are presented in Section 3 and summarized in Section 4.

\section{Computational model for MD simulation of PLAL}

The simulations reported in this paper are performed with a hybrid computational model combining a coarse-grained representation of liquid (parameterized for water), a fully atomistic description of laser interactions with metal targets, and acoustic impedance matching boundary conditions designed to mimic the non-reflecting propagation of the laser-induced pressure waves through the boundaries of the computational domain. A schematic representation of the computational system is shown in Fig. 1. The simulations are performed for thin Ag film deposited on a transparent silica substrate, covered by water, and irradiated by a femtosecond laser pulse from the side of the transparent substrate. A brief description of the main components of the computational model as well as details of the computational setup are provided below.

\subsection{TTM-MD model for laser interactions with metals}

The laser interaction with the metal film is simulated with a hybrid atomistic-continuum model [62-65] that combines the classical molecular dynamics (MD) method with twotemperature model (TTM) [66]. The TTM describes the evolution of lattice and electron temperatures in metals by two coupled nonlinear differential equations that account for the laser excitation of the conduction band electrons, energy exchange between the electrons and atomic vibrations due to the electron-phonon coupling, and electron heat conduction. In the combined TTM-MD model, MD substitutes the TTM equation for the lattice temperature, the cells of the three-dimensional (3D) finite difference discretization grid used in the solution of the TTM equation for the electron temperature are mapped to the corresponding volumes of the MD system, and the local lattice temperature is defined for each cell from the average kinetic energy of thermal motion of atoms. The electron temperature enters a coupling term added to the MD equations of motion to account for the energy exchange between the electrons and the lattice [62]. Note that, following the terminology established in the literature presenting TTM calculations, the term "lattice temperature" does not imply the preservation of the crystalline order in the 
irradiated material but is simply used here to refer to the temperature of the ionic subsystem that is brought out of equilibrium with the conduction-band electrons. A complete description of the TTM-MD model is provided elsewhere [62], and below we only provide the parameters of the model used in the simulations reported in this paper.

The interatomic interactions in the MD part of the model are described by the embedded atom method (EAM) potential with the functional form and parameterization developed in Ref [67]. A cut-off function suggested in Ref. [68] is added to the potential to smoothly bring the interaction energies and forces to zero at interatomic distance of $5.5 \AA$. Although the potential is fitted to low-temperature values of the equilibrium lattice constant, sublimation energy, elastic constants, and vacancy formation energy, it also provides a good description of high-temperature thermodynamic properties of $\mathrm{Ag}$ [69] relevant to the simulation of laser-induced processes. In particular, the equilibrium melting temperature, $T_{m}$, determined in liquid-crystal coexistence simulations, is $1139 \pm 2 \mathrm{~K}$ [70], about $8 \%$ below the experimental values of $1235 \mathrm{~K}$ [71]. The threshold temperature for the onset of the explosive phase separation into liquid and vapor, $T^{*}$, determined in simulations of slow heating of a metastable liquid, is found to be $\sim 3450 \mathrm{~K}$ at zero pressure and $\sim 4850 \mathrm{~K}$ at $0.5 \mathrm{GPa}$ [72]. The onset of the phase explosion can be expected at $10 \%$ below the critical temperature [73-75] and the values of $T^{*}$ calculated for the EAM Ag material are not in conflict with the range of experimental values of the critical temperature of $\mathrm{Ag}$ spanning from $4300 \mathrm{~K}$ to $7500 \mathrm{~K}[76]$.

The electron temperature dependences of the thermophysical material properties included in the TTM equation for the electron temperature (electron-phonon coupling factor and electron heat capacity) are taken in the forms that account for the thermal excitation from the electron states below the Fermi level [77]. The electron thermal conductivity is described by the Drude model relationship, $K_{e}\left(T_{e}, T_{l}\right)=v^{2} C_{e}\left(T_{e}\right) \tau_{e}\left(T_{e}, T_{l}\right) / 3$, where $C_{e}\left(T_{e}\right)$ is the electron heat capacity, $v^{2}$ is the mean square velocity of the electrons contributing to the electron heat conductivity, approximated in this work as the Fermi velocity squared, $v_{\mathrm{F}}^{2}$, and $\tau_{e}\left(T_{e}, T_{l}\right)$ is the total electron scattering time defined by the electron-electron and electron-phonon scattering rates, $1 / \tau_{e}=1 / \tau_{e-e}+1 / \tau_{e-p h}=A T_{e}^{2}+B T_{l}$. The value of the coefficient $A, 3.57 \times 10^{6} \mathrm{~s}^{-1} \mathrm{~K}^{-2}$, is estimated within the free electron model, following the approach suggested in [78]. The value of 
the coefficient $B, 1.12 \times 10^{11} \mathrm{~s}^{-1} \mathrm{~K}^{-1}$ is obtained from the experimental value of the thermal conductivity of solid Ag at the melting temperature, $363 \mathrm{Wm}^{-1} \mathrm{~K}^{-1}$ [79].

The implementation of the model used in this work does not account for ionization of the ejected plume, as simple estimations based on the Saha-Eggert equation $[80,81]$ suggest that the degree of ionization in the ablation plume is negligible under irradiation conditions applied in the simulations reported in this paper. As a result, the nanoparticle formation through nucleation around ion seeds in "misty plasma" considered for high fluence nanosecond PLAL $[48,82]$ is not relevant to the milder irradiation conditions and short pulses considered in the present work.

\subsection{Coarse-grained MD representation of liquid environment}

The direct application of the conventional all-atom MD representation of liquids in largescale simulations of laser processing or ablation is not feasible due to the high computational cost. Thus, a coarse-grained representation of the liquid environment [56,83], where each particle represents several molecules, is adapted in this work. The coarse-graining reduces the number of degrees of freedom that have to be treated in the MD simulations and significantly increases the time and length scales accessible for the simulations. At the same time, however, the smaller number of the dynamic degrees of freedom results in a severe underestimation of the heat capacity of the liquid. To resolve this problem, the degrees of freedom that are missing in the coarse-grained model are accounted for through a heat bath approach that associates an internal energy variable with each coarse-grained particle [84-86]. The energy exchange between the internal (implicit) and dynamic (explicit) degrees of freedom are controlled by the dynamic coupling between the translational degrees of freedom and the vibrational (breathing) mode associated with each coarse-grained particle (the particles are allowed to change their radii, or to "breath" $[83,86])$. The energy exchange is implemented through a damping or viscosity force applied to the breathing mode, which connects it to the energy bath with capacity chosen to reproduce the real heat capacity of the group of atoms represented by each coarse-grained particle. Effectively, the breathing mode serves as a "gate" for accessing the energy stored in the molecular heat bath.

The first implementation of the coarse-grained model with heat bath approach was recently developed for water and applied to simulations of laser interactions with water-lysozyme system [86]. Each coarse grained particle in the model has a mass of $50 \mathrm{Da}$ and represents about three 
real water molecules. The potential describing the inter-particle interactions is provided in Ref. [86] and the parameters of the potential are selected to ensure a satisfactory semi-quantitative description of experimental properties of water. While one cannot expect the coarse-grained model to provide an accurate representation of all the structural and thermodynamic properties of water, the key physical properties predicted by the model, such as density, speed of sound, bulk modulus, viscosity, surface energy, melting temperature, critical temperature, and critical density, are found to not deviate from the experimental values by more than $25 \%$, as can be seen from Table 1. The room temperature density, $\rho$, and heat capacity, $c_{p}$, are fitted exactly through the selection of the equilibrium inter-particle distance and the capacity of the heat bath associated with each coarse-grained particle. The bulk modulus, $K$, is calculated from the pressure-volume dependence obtained in MD simulations and the speed of sound, $c_{s}$, is evaluated from the values of $K$ and $\rho$. The melting temperature of the coarse grained "water", $T_{m}$, is determined in a constant-pressure and constant-energy liquid-crystal coexistence simulation and is about $21 \%$ higher than the experimental value. The critical temperature and density, $T_{c}$ and $\rho_{c}$, are extrapolated from the results of constant-temperature and constant-pressure liquid-gas coexistence simulation from 300 to $500 \mathrm{~K}$ following the method of Ref. [87]. Note that the critical temperature listed in Table 1 is slightly lower than the value roughly estimated from the threshold temperature for the phase explosion and provided in Ref. [86]. Thermal conductivity, $k$, is evaluated by fitting the evolution of the temperature profiles in an elongated MD system with a heat source applied in the middle to the solution of the heat diffusion equation. The dynamic viscosity, $\eta$, is calculated with the Stoke-Einstein equation, using the values of the selfdiffusion coefficient evaluated from the time dependence of the mean square displacement of coarse-grained particles in a MD simulation performed at $300 \mathrm{~K}$. Finally, the surface energy, $\gamma$, is obtained based on the results of a liquid-gas coexistence simulation performed at $300 \mathrm{~K}$.

The interactions between Ag atoms and the coarse-grained water particles are described by the Lennard-Jones (LJ) potential with the following length and energy parameters: $\sigma=1.92 \AA$ and $\varepsilon=0.145 \mathrm{eV}$. The parameters are fitted to match the diffusion of metal atoms and small clusters in water predicted by the Stoke-Einstein equation at $300 \mathrm{~K}$ and calculated in constanttemperature and constant-volume MD simulations. Furthermore, the values of $\sigma$ and $\varepsilon$ are chosen to ensure that the values of the equilibrium $\mathrm{O}-\mathrm{Ag}$ distance and the adsorption energy of water on a Ag surface predicted in ab-initio simulations [88-91] are roughly reproduced in the 
coarse-grained model. Note that, while it is possible to incorporate the description of chemical reactions into the framework of coarse-grained MD model [56,92,93], we have not included description of oxidation or other chemical reactions [94] in the version of the model used in the present study.

\subsection{Computational setup and simulation parameters}

The simulations are performed for a $20 \mathrm{~nm} \mathrm{Ag}$ film deposited on a transparent silica substrate and covered by water, as shown in Fig. 1. The initial film has fcc crystal structure and (001) orientation of the free surface. The periodic boundary conditions are applied in the directions parallel to the surface of the film and the dimensions of the computational system in these directions are $100 \mathrm{~nm} \times 100 \mathrm{~nm}$.

The silica substrate is not represented with atomic resolution but introduced through a dynamic acoustic impedance matching boundary condition that accounts for the displacement of the metal-substrate interface in response to the thermal expansion and phase transformations occurring in the metal film. This boundary condition is designed to reproduce the transformation of a part of the energy deposited by the laser pulse into the energy of a pressure generated in the substrate, as well as the work of adhesion between the silica substrate and Ag film. The details of the implementation and parametrization of the boundary condition are described in Ref. [72].

The water environment above the Ag film is represented by a combination of the coarsegrained MD model described above, in Section 2.2, and a dynamic acoustic impedance matching boundary condition $[72,95,96]$ that ensures non-reflective propagation of the pressure wave generated at the metal-water interface into the bulk of the water environment. This boundary condition is suitable for simulation of experimental conditions where reflection of the pressure wave from the surface of a thick water overlayer does not play any significant role in the generation of nanoparticles. The coarse-grained MD representation is used in a 300-nm-thick region of the water environment adjacent to the $\mathrm{Ag}$ film and the dynamic boundary condition is applied at the top of this region.

The computational system consists of 11.3 million Ag atoms and 36.1 million coarse-grained particles representing water environment. The system is equilibrated at $300 \mathrm{~K}$ for 200 ps before 
applying laser irradiation. The simulations are performed with a computationally-efficient parallel code implementing the combined TTM-MD - coarse-grained MD model.

The irradiation of the target with a 40 fs laser pulse is represented through a source term added to the equation for the electron temperature [62]. The source term simulates excitation of the conduction band electrons by a laser pulse with a Gaussian temporal profile and reproduces the exponential attenuation of laser intensity with depth under the surface. The optical absorption depth, $12 \mathrm{~nm}$ at laser wavelength of $800 \mathrm{~nm}$ [97], combined with the effective depth of the "ballistic" energy transport, estimated to be about $56 \mathrm{~nm}$ for $\mathrm{Ag}[65,72]$, is used in the source term of the TTM equation $[62,98]$. Since the ballistic range in Au exceeds the thickness of the films considered in this work, $20 \mathrm{~nm}$, the reflection of the ballistic electrons from the back surface of the film results in a uniform distribution of the electronic temperature established on the time scale of electron thermalization. The effect of the ballistic energy transport and the finite size of the film are accounted for in the source term describing the laser irradiation [62,98], leading to an almost uniform energy deposition throughout the film thickness. The reflectivity of the surface is not defined in the model since the absorbed laser fluence rather than the incident fluence is used in the presentation of the simulation results.

\section{Results and discussion}

In this section, we report the results of two large-scale MD simulations of laser ablation of thin Ag films in liquid environment. Series of snapshots of atomic configurations generated in the course of the MD simulations are supplemented by the analysis of the evolution of thermodynamic parameters in response to the laser energy deposition and are used in the discussion of the characteristic features of laser ablation in liquids in Sections 3.1 and 3.2. The distinct mechanisms of the nanoparticle formation in PLAL revealed in the simulations and the connection between the computational predictions and experimental observations are discussed in Section 3.3.

\subsection{Visual picture of laser ablation in liquid}

The simulations discussed in this section are performed for $20 \mathrm{~nm} \mathrm{Ag} \mathrm{films} \mathrm{irradiated} \mathrm{at}$ absorbed laser fluences of 400 and $700 \mathrm{~J} / \mathrm{m}^{2}$. For irradiation in vacuum [99], these fluences correspond to the regime of phase explosion [63,64,73-75], when the entire Ag film undergoes 
an explosive decomposition into liquid droplets and vapor. The threshold for the phase explosion in vacuum is determined to be $310 \mathrm{~J} / \mathrm{m}^{2}$ for a $20 \mathrm{~nm}$ Ag film in a separate series of simulations. To provide a reference for the analysis of the effect of liquid environment on the ablation dynamics, snapshots of atomic configurations and a density contour plot are shown in Fig. 2 for a simulation performed for a $20 \mathrm{~nm} \mathrm{Ag} \mathrm{film} \mathrm{irradiated} \mathrm{at} \mathrm{an} \mathrm{absorbed} \mathrm{fluence} \mathrm{of} 360$ $\mathrm{J} / \mathrm{m}^{2}$ in vacuum. At this fluence, the laser excitation and rapid equilibration between the excited electrons and lattice vibrations (electron-phonon coupling [77]) overheats the film material above the limit of its thermodynamic stability [73-75], leading to an explosive release of vapor and ejection of a mixture of vapor and small liquid droplets. The ejected vapor-droplet mixture quickly expands away from the substrate, as can be seen from the snapshots in Fig. $2 \mathrm{a}$ and the rapidly dropping density in Fig. 2 b.

The dynamics of the ablation plume expansion is strongly altered by the presence of the liquid environment, as can be seen from Figs. 3 and 4, where two series of snapshots of atomic configurations produced in the simulations of PLAL are presented along with normalized density distributions plotted for both silver and water. Even though the absorbed laser fluences in these simulations are higher than that in the simulation illustrated by Fig. 2, the expansion of a hot mixture of vapor and liquid droplets generated in the phase explosion of the superheated $\mathrm{Ag}$ film is meeting a stiff resistance from the water environment and is largely suppressed. While the expansion is stronger at $700 \mathrm{~J} / \mathrm{m}^{2}$, in both simulations the products of the film decomposition are rapidly decelerating and remain confined within hundreds of nanometers from the substrate during the first nanoseconds after the laser irradiation.

The inspection of the density profiles plotted to the right from the snapshots in Figs. 3 and 4 reveals the formation of a dense front of the Ag plume near the interface with water by the time of 200 ps, with a sharper density peak observed in the simulation performed at a lower fluence of $400 \mathrm{~J} / \mathrm{m}^{2}$. The density values in the layers at the front of the expanding plume correspond to a strongly superheated liquid metal that can be expected to exhibit a limited stability as it interacts with compressed water layer. Indeed, the snapshots from the simulations show that the morphologies of the dense superheated layers (green layers in Figs. 3 and 4) undergo continuous evolution throughout the simulations. The fine roughness of the top surfaces of the layers exposed to the water environment can be seen already at $200 \mathrm{ps}$ and is particularly pronounced in the simulation performed at the higher fluence, Fig. 4. The interfacial structures coarsen and 
decompose into large liquid regions on the timescale of nanoseconds. The important implications of the liquid layer decomposition for the nanoparticle formation are discussed in Section 3.3.

Another prominent process that plays an important role in the nanoparticle generation and, as discussed in Section 3.2, can be related to experimental observations of large, micrometers to millimeters, bubbles in PLAL [37,42-48], is the appearance and growth of a low-density Agwater mixing region at the interface between the dense layer of the superheated liquid metal and the water environment. While in both simulations the initial fronts of the density profiles are relatively sharp and the metal-water mixing region does not exceed $30 \mathrm{~nm}$ at $200 \mathrm{ps}$, the mixing region steadily expands with time and reaches the thickness of about $100 \mathrm{~nm}$ by the time of $3 \mathrm{~ns}$ after the laser pulse. As discussed below, the expansion of the mixing region is driven by the rapid heating and vaporization of water that is brought in contact with the hot mixture of metal vapor, clusters, and droplets produced by the explosive decomposition of the irradiated film. The thermodynamic conditions in the mixing region are highly susceptible to the nucleation and growth of metal clusters and nanoparticles, which is clearly reflected in the snapshots, where a large number of small clusters can be seen to emerge in the mixing region on the timescale of just a couple of nanoseconds.

\subsection{Evolution of thermodynamic parameters}

The analysis of the visual picture of the ablation process can be supplemented by consideration of the evolution of density, temperature, pressure, and collective velocity in the direction away from the substrate, shown in the form of contour plots in Figs. 5 and 6 . The initial rapid expansion of the irradiated film undergoing an explosive decomposition into vapor and small droplets, the formation of a dense layer of hot metal plume pushing against the water environment, as well as the appearance and expansion of a low-density metal-water mixing region are the common features of the two simulations that can be seen in the contour plots. To facilitate the analysis, several distinct regions can be identified in the contour plots based on the evolving distributions of $\mathrm{Ag}$ and water. These regions are labeled in the density contour plots, Figs. 5a and 6a, and are defined and discussed below.

The first region is a low density region that appears in the vicinity of the silica substrate as a result of the rapid expansion of the metal film undergoing the phase explosion. It is defined as a 
region where the density of the products of the explosive film decomposition (a mixture of $\mathrm{Ag}$ vapor, atomic clusters, and small droplets) is below $20 \%$ of the initial density of the metal film. The second region is a dense part of the ablation plume that is generated due to the confinement of the plume expansion by the water environment. The interaction of this dense layer with water results in the formation of a complex protruding morphology of the top surface of the dense layer that can be seen in the snapshots in Figs. 3 and 4. The layer, therefore, can be subdivided into a more compact/uniform part (region 2) and a "terrain" region (region 3) where the liquid metal protrusions and large droplets are surrounded by supercritical water. The boundary between the second and third regions is defined as the location where the concentration of water exceeds 0.5 wt. $\%$ in the simulation performed at a fluence of $400 \mathrm{~J} / \mathrm{m}^{2}$ and $5 \mathrm{wt} . \%$ in the one performed at $700 \mathrm{~J} / \mathrm{m}^{2}$. The upper boundary of the terrain region is also defined by the water concentration and corresponds to 5 and 24 wt.\% water in the lower and higher fluence simulations, respectively. The different thresholds used for identification of the second and third regions in the two simulations are chosen to match the visual identification of the "compact" and "terrain" parts of the dense metal layer from the simulation snapshots, Figs. 3 and 4, and are reflecting the stronger penetration of water into the layer at a higher fluence.

Above the "terrain" region, we observe an expanding low-density region (region 4 in the contour plots) where Ag atoms, clusters, and nanoparticles are suspended in water that is brought to the supercritical state by the interaction with the hot metal plume. The density of water in the lower part of this region is below 0.1 and $0.2 \mathrm{~g} / \mathrm{cm}^{3}$ in the simulations performed at $400 \mathrm{~J} / \mathrm{m}^{2}$ and $700 \mathrm{~J} / \mathrm{m}^{2}$, respectively, and the boundaries of the region are defined so that the concentration of water is ranging from 5 to $98 \mathrm{wt} . \%$ at $400 \mathrm{~J} / \mathrm{m}^{2}$, and from 24 to $98 \mathrm{wt} . \%$ at $700 \mathrm{~J} / \mathrm{m}^{2}$. Finally, the fifth region is defined as a water region where the concentration of $\mathrm{Ag}$ is below $2 \mathrm{wt} \%$ and the water remains close to its initial density of $1 \mathrm{~g} / \mathrm{cm}^{3}$.

The most distinctive difference between the evolutions of different regions in the two simulations reported in this paper is the dynamics of the dense Ag layer formed at the interface with the water environment (regions 2 and 3). At a lower fluence of $400 \mathrm{~J} / \mathrm{m}^{2}$, the dense liquid layer starts to drop down after $500 \mathrm{ps}$ and redeposits to the substrate at $\sim 1150 \mathrm{ps}$. At a higher fluence of $700 \mathrm{~J} / \mathrm{m}^{2}$, the molten layer is also decelerated by the resistance of the water environment but continues to float above the substrate on the timescale of the simulation. The difference in the layer dynamics can be explained by considering the difference in the 
thermodynamic conditions in the regions immediately adjacent to the superheated liquid layer, i.e., in regions 1 and 4. As can be seen from the temperature contour plots, the levels of temperature and pressure in region 1 are substantially higher in the simulation performed at a higher fluence (note the difference in scales used for temperature and pressure in Figs. 5 and 6). As a result, the force exerted on the layer from the expanding region 1 is weaker in the lower fluence simulation and is quickly exceeded by the counterforce applied on the layer from region 4, where the expansion of heated supercritical water is fueled by the rapid energy transfer from the hot metal plume. The expanding Ag-water mixing region is able in this case to push the dense front of the plume down to the substrate while simultaneously pushing the overlaying water region in the upward direction. At a higher fluence of $700 \mathrm{~J} / \mathrm{m}^{2}$, a similar downward push from the emerging Ag-water mixing region meets stiffer resistance from the underlying gaseous region 1 , where the emission and reflection of a compressive wave can be seen in the pressure and velocity contour plots shown in Figs. 6c and 6d. As a result, the position of the layer undergoes a series of weak oscillations while floating above the silica substrate.

\subsection{Nanoparticle formation mechanisms}

The results of the simulations discussed above make it possible to identify two distinct mechanisms of nanoparticle formation in laser ablation of metals in water environment. Both mechanisms are activated by the formation of the dense layer of superheated liquid metal at the water-plume interface at the early stage of the ablation plume expansion. The interaction of water with the hot metal layer brings an interfacial region of water to the supercritical state and results in the formation of a low density metal-water mixing region that rapidly expands and serves as a precursor for the formation of cavitation bubble at a later time. As the evaporation provides the main pathway for the hot molten layer to cool down, the layer serves as a continuous source of metal vapor supplied to the mixing region. The conditions in the mixing region are highly susceptible to the condensation of metal atoms into clusters and rapid growth of the atomic clusters into nanoparticles. Indeed, it can be seen from the contour plots in Figs. $5 \mathrm{~b}$ and $6 \mathrm{~b}$ that the average temperature in the mixing regions, while staying above the critical temperature of water, is close to and, in the upper part, even below the melting temperature of Ag. The rapid nucleation and growth of the nanoparticles is initiated on the very short timescale of just a few nanoseconds after the laser irradiation and is directly observed in the MD 
simulations. This constitutes the first nanoparticle generation mechanism predicted in the simulations, and further analysis of the kinetics of the nanoparticle formation through this mechanism is provided below.

The analysis of the evolution of sizes of the metal clusters and nanoparticles in the mixing region is performed with a cluster identification algorithm applied to atomic configurations generated in the two simulations between 300 ps and 3000 ps, with a 100 ps interval. Only the clusters detected above the molten layer (regions 3 and 4) are considered in the analysis, since these are the clusters that contribute to the generation of nanoparticles in the mixing region. The results of the analysis are presented in Figs. 7 and 8, where the cumulative numbers of $\mathrm{Ag}$ atoms present above the molten layer as individual atoms and small atomic clusters with diameter below $1 \mathrm{~nm}$ (less than 30 atoms) are shown separately from the larger clusters that we denote as nanoparticles. As the total number of atoms in the mixing region is steadily rising due to the evaporation from the superheated liquid layer, the number of individual atoms and small atomic clusters saturates in both simulations, Figs. 7a and 8a. This reflects the high rate of the nanoparticle growth that depletes the population of atoms and clusters faster than they are resupplied by the layer evaporation.

The evolution of the abundance of nanoparticles of different sizes is shown in Figs. 7b and $8 \mathrm{~b}$. The populations of nanoparticles with diameters less than 4-6 $\mathrm{nm}$ seem to plateau and even show signs of decline in the lower fluence simulation, Fig. 7b, as the smaller nanoparticles grow and coalesce into the larger ones. Indeed, the larger nanoparticles keep up the trend of cumulative number of atoms in the mixing region growing when the abundance of smaller nanoparticles go flat. As can be seen from the snapshots in Figs. 3 and 4, the larger nanoparticles are mostly found closer to the molten layer, where the higher density of atoms and other nanoparticles leads to the faster growth and higher probability of nanoparticle coalescence. Overall, the nanoparticle size distribution shifts to larger sizes as time progresses and nanoparticles grow and coalesce. The growth of the nanoparticles and broadening of the size distributions is further visualized by plotting the histograms of the amount of material contributing to nanoparticles of different sized at three moments of time, 1, 2, and 3 ns after the laser pulse, Figs. 7c and 8c. 
While the simulation performed at a fluence of $700 \mathrm{~J} / \mathrm{m}^{2}$ has stronger plume expansion, the subsequent vapor and clusters growth above the molten layer is substantially slower than that at $400 \mathrm{~J} / \mathrm{m}^{2}$. This observation can be related to the additional contribution to the rapid expansion of the mixing region coming in the lower fluence simulation from the downward motion and redeposition of the liquid layer. The wider mixing region facilitates the evaporation of the liquid layer that produces more than twice higher total number of $\mathrm{Ag}$ atoms as compared to the simulation performed at $700 \mathrm{~J} / \mathrm{m}^{2}$, Figs. 7a and $8 \mathrm{a}$. At the same time, the mixing region generated at a fluence of $700 \mathrm{~J} / \mathrm{m}^{2}$ has a pressure of $0.064 \mathrm{GPa}$ at the end of simulation, as compared $0.025 \mathrm{GPa}$ in the same region in the lower fluence simulation. The difference in the values of pressure indicate that further expansion is likely to be more extensive at the higher fluence, which may lead to a higher final nanoparticle yield.

One important aspect of the nanoparticle generation mechanism predicted in the simulations is the rapid quenching of the metal vapor ejected into the water-metal mixing region. While the interaction between the hot metal vapor and water brings the water to the supercritical state, the same interaction cools the metal vapor and nanoparticles down to the temperature that can be sufficiently low to cause solidification of the nanoparticles. As one can observe from Figs. 3 and 4, the nanoparticles in the top part of the mixing region are colored blue, indicating the low level of potential energy. To clearly show the temperature of the nanoparticles generated by the end of the simulations, the final snapshots colored by local temperature are provided in Fig. 9. While the nanoparticles located closer to the superheated liquid layer are green and have temperatures that are about twice the melting temperature of $\mathrm{Ag}$, marked as $T_{m}$ in the temperature scale, the small blue nanoparticles in the top part of the mixing region are already below $T_{m}$ and can be expected to be solid. Indeed, the enlarged views of two representative "blue" nanoparticles shown in Fig. 9 clearly demonstrate their (poly)crystalline structure. While the observation of the crystalline structure of $\mathrm{Ag}$ nanoparticles generated in the simulations is consistent with experimental results reported for PLAL of Ag targets [100], the surprising prediction of the simulations is that the cooling of the metal vapor injected into the mixing region from the phase explosion temperature generated in the $\mathrm{Ag}$ film by the laser excitation down to the melting temperature of $\mathrm{Ag}$ takes less than $3 \mathrm{~ns}$, resulting in the effective cooling rate on the order of $10^{12}$ $\mathrm{K} / \mathrm{s}$ and producing solid nanoparticles at the very early stage of the ablation process. The 
ultrafast growth and quenching rates may result in the generation of nanoparticles with highly nonequilibrium metastable structures and phases.

The second mechanism of the nanoparticle generation that can be inferred from the simulation results is the disintegration of the superheated liquid layer itself, which can directly yield much larger, on the order of tens of nanometers, nanoparticles. While the timescale of the complete breakup of the liquid layer exceeds the timescales accessible to MD simulations, the first manifestation of this nanoparticle generation mechanism can be observed in the simulation snapshots, Figs. 3 and 4, where the morphology of the "terrain" region at the vapor-plume interface is gradually evolving towards the formation of individual large droplets. Indeed, the first droplet produced through this mechanism can already be observed in the last snapshot shown in Fig. 4. Although the temperature of this droplet, that consist of 354,455 atoms and has diameter of $22.8 \mathrm{~nm}$, is more than twice the melting temperature of $\mathrm{Ag}$ at the time of its separation from the liquid layer (see Fig. 9), the rapid cooling in the mixing region can be expected to result in its eventual freezing into a solid nanoparticle.

The decomposition of the thin superheated liquid layer into individual droplets may, in principle, be driven by the inherent thin film instability at high temperatures [64,101], although the results of the simulations suggest that the interaction of the metal layer with the compressed supercritical water is playing the dominant role in defining the morphology to the "terrain" region and, eventually, the characteristic sizes of nanoparticles generated by the film breakup. The appearance of roughness at the interface between the metal layer and the supercritical water can be observed as early as 200 ps after the laser pulse, Figs. 3 and 4, and the interfacial morphology evolve rapidly as the metal layer is decelerated and, in the lower fluence simulation, pushed back to the substrate by the expansion of the supercritical water region during the first nanosecond of the ablation process. The development of the complex morphology of the interface between the higher density metal layer decelerated by the pressure from the lighter supercritical water (see Figs. 5a and 6a) can be attributed to the Rayleigh-Taylor instability of the interface between the accelerated fluid layers [102,103]. The morphological features of the interfacial region are finer in the higher fluence simulation (Fig. 4) as compared to the ones at the lower fluence (Fig. 3), which is related to more active initial mixing and lower viscosity at higher temperature leading to the higher spatial frequency of the interfacial features. Although the quantitative aspects of the nanoparticle generation through the breakup of a laser-generated 
superheated liquid induced by the Rayleigh-Taylor instability are still being investigated and will be reported elsewhere, this mechanism appears to be general (observed in the simulation of PLAL of bulk targets) and can be utilized for controlled nanoparticle generation and surface nanostructuring by laser ablation in liquid environment.

The computational prediction of the existence of two distinct mechanisms of nanoparticle formation in PLAL is consistent with experimental observations of bimodal nanoparticle size distributions $[4,5]$ and can be related to the results of recent time-resolved SAXS probing of the cavitation bubble dynamics [43-45], where two groups of nanoparticles with different characteristic sizes have been observed to emerge at different stages of the bubble evolution. The experimental observation of "primary particles," with the size distribution centered around 8-10 $\mathrm{nm}$, can be related to the nanoparticles generated by the rapid condensation and growth in the expanding mixing region in the simulations. Indeed, the presence of the primary particles at the earliest stage of the cavitation bubble expansion, the higher population of the nanoparticles in the lower part of the bubble and the decrease of the nanoparticle abundance and size with increasing distance from irradiated surface are the experimental observations that are consistent with the nucleation and growth pathway of the nanoparticle formation predicted in the simulations.

The second group of nanoparticles, the so-called "secondary particles" with the size distribution of around $30-50 \mathrm{~nm}$, are observed to appear at a later stage of the cavitation bubble evolution and are speculated to form through agglomeration of the primary particles during the bubble collapse [43-45]. While the MD simulations cannot provide information on the whole cavitation bubble dynamics, they offer an alternative plausible explanation of the origin of the secondary particles. The simulations predict that the secondary particles may appear through the formation of a dense layer of superheated liquid metal at the front of the ablation plume confined by the water environment, followed by disintegration of the molten layer facilitated by its interaction with the compressed supercritical water. The collapse of the bubble may simply disperse the large nanoparticles already formed through the molten metal layer disintegration, or it can actually cause the breakdown of the molten layer if it survives until this late stage of the cavitation bubble dynamics. 
While the simulations reported in this paper are performed for thin films, the discussion of the mechanisms of the nanoparticle generation revealed in the simulations is fully applicable to the ablation of bulk targets in a liquid environment. The first simulations performed for bulk targets, to be reported elsewhere, show that the dynamics of the ablation plume generated through the explosive disintegration of a top layer of the irradiated bulk target is very similar to the one discussed above for the thin film ablation. The confinement of the ablation plume by the water environment results in the formation of a dense layer of superheated metal at the waterplume interface, followed by the appearance and expansion of a metal - supercritical water mixing region and generation of two populations of nanoparticles through the condensation in the mixing region and disintegration of the liquid layer.

The results are also not specific for the femtosecond laser pulses, as the increase of the lattice temperature in the film is controlled by the timescale of the electron-phonon equilibration, $\tau_{e-p h} \sim$ $8 \mathrm{ps}$, defined as the time constant of the exponential decay of the energy of the excited electrons [72]. This time is comparable to the time needed for the mechanical relaxation (expansion) of the film, $\tau_{m} \sim L_{f} / C_{s} \approx 5.5 \mathrm{ps}$, where $L_{f}=20 \mathrm{~nm}$ is the film thickness and $C_{s} \approx 3650 \mathrm{~m} / \mathrm{s}$ is the speed of sound in Ag. As a result, the heating takes place under conditions of partial stress confinement [62-65,99], leading to a stronger initial expansion of the material undergoing the phase explosion $[63,104]$. Any pulse duration that is shorter than $\tau_{e-p h}$ would produce results that very similar to the ones reported in this paper. The increase of the pulse duration above $\tau_{e-p h}$ would allow for the film expansion during the laser heating, thus diminishing the contribution of photomechanical effects to the ejection of the plume and generation of nanoparticles.

\section{Summary}

An advanced computational model combining an atomistic description of laser interactions with metal targets, a coarse-grained representation of liquid (parameterized for water), and acoustic impedance matching boundary conditions designed for representation of a transparent substrate (parameterized for silica glass) and non-reflecting propagation of laser-induced pressure wave in the liquid environment is developed and applied for investigation of the mechanisms of nanoparticle generation in PLAL. First atomistic simulations of laser ablation of thin $\mathrm{Ag}$ films in water environment have provided detailed microscopic information on the 
unique characteristics of laser ablation in liquids and revealed two distinct mechanisms of nanoparticle formation in PLAL.

In contrast to the laser ablation in vacuum or a background gas, where nanoparticles can be directly produced via explosive decomposition of the superheated target material into a mixture of vapor, atomic clusters and droplets, the interaction of the ablation plume with liquid environment leads to a rapid deceleration of the ejected material and the formation of a dense superheated molten layer at the water-plume interface. The water in contact with the hot metal layer is brought to the supercritical state and transforms into expanding low density metal-water mixing region that serves as a precursor for the formation of a cavitation bubble at a later time. The conditions in the mixing region facilitate condensation of metal atoms into clusters and rapid growth of the atomic clusters into nanoparticles with maximum sizes barely exceeding $10 \mathrm{~nm}$. The interaction of the growing nanoparticles with supercritical water provides a highly efficient cooling mechanism that produces an effective cooling rate for the metal vapor and clusters of up to $10^{12} \mathrm{~K} / \mathrm{s}$ and results in solidification of nanoparticles located in the upper part of the mixing region on the timescale of nanoseconds.

In addition to the nanoparticles produced by the rapid nucleation and growth in the watermetal mixing region, the second mechanism of the nanoparticle generation identified in the simulations is the breakup of the layer of superheated molten metal accumulated at the plumewater interface. The preliminary analysis of the initial stage of the layer decomposition suggests that the emergence of complex morphological features in the interfacial region can be attributed to the Rayleigh-Taylor instability of the interface between the higher density metal layer decelerated by the pressure from the lighter supercritical water. The nanoparticles produced through the disintegration of the superheated liquid layer are larger than the ones produced through the condensation in the mixing region and have characteristic sized on the order of tens of nanometers.

The computational prediction of the two mechanisms of the nanoparticle generation yielding nanoparticles with different characteristic sizes is consistent with experimental observations of the bimodal nanoparticle size distributions produced by PLAL and can be related to the results of recent time-resolved SAXS probing of the cavitation bubble dynamics, where the appearance of two different nanoparticle populations is detected at different stages of the bubble evolution. 


\section{Acknowledgments}

Financial support for this work was provided by the National Science Foundation (NSF) through Grants CMMI-1301298 and CMMI-1436775, as well as by Austrian Science Fund (FWF) through the Lise Meitner Programme (project M 1984). Computational support was provided by the Oak Ridge Leadership Computing Facility (INCITE project MAT130) and NSF through the Extreme Science and Engineering Discovery Environment (Project TG-DMR110090). 


\section{References}

1. A. Fojtik and A. Henglein, Laser ablation of films and suspended particles in a solvent: formation of cluster and colloid solutions, Ber. Bunsenges. Phys. Chem. 97, 252-254 (1993)

2. F. Mafune, J-Y. Kohno, Y. Takeda, and T. Kondow, Formation of gold nanoparticle by laser ablation in aqueous solution of surfactant, J. Phys. Chem. B 105, 5114-5120 (2000)

3. G. Compagnini, A. A. Scalisi, and O. Puglisi, Ablation of noble metals in liquids: a method to obtain nanoparticles in a thin polymeric film, Phys. Chem. Chem. Phys. 4, 2787-2791 (2002)

4. A. V. Kabashin and M. Meunier, Synthesis of colloidal nanoparticle during femtosecond laser ablation of gold in water, J. Appl. Phys. 94, 7941-7943 (2003)

5. J.-P. Sylvestre, A. V. Kabashin, E. Sacher, and M. Meunier, Femtosecond laser ablation of gold in water: influence of the laser-produced plasma on the nanoparticle size distribution, Appl. Phys. A 80, 753-758 (2005)

6. S. Barcikowski, F. Devesa, K. Moldenhauer, Impact and structure of literature on nanoparticle generation by laser ablation in liquids, J. Nanopart. Res. 11, 883-1893 (2009)

7. R. Streubel, S. Barcikowski, and B. Gökce, Continuous multigram nanoparticle synthesis by high-power, high-repetition-rate ultrafast laser ablation in liquids, Opt. Lett. 41, 14861489 (2016)

8. R. Streubel, G. Bendt, and B. Gökce, Pilot-scale synthesis of metal nanoparticles by highspeed pulsed laser ablation in liquids, Nanotechnology 27, 205602 (2016)

9. C. Hess, A. Schwenke, P. Wagener, S. Franzka, C. L. Sajti, M. Pflaum, B. Wiegmann, A. Haverich, and S. Barcikowski, Dose-dependent surface endothelializationand biocompatibility of polyurethane noble metal nanocomposites, J. Biomed. Mater. Res. A 102, 1909-1920 (2014)

10. S. Petersen and S. Barcikowski, In situ bioconjugation: Single step approach to tailored nanoparticle-bioconjugates by ultrashort pulsed laser ablation, Adv. Funct. Mater. 19, 1167-1172 (2009)

11. V. L. Kumar, R. S. S. Siddhardha, A. Kaniyoor, R. Podila, M. Molli, S. M. V. Kumar, K. Venkataramaniah, S. Ramaprabhu, A. M. Rao, and S. S. Ramamurthy, Gold decoratedgraphene by laser ablation for efficient electrocatalytic oxidation of methanoland ethanol, Electroanalysis 26, 1850-1857 (2014)

12. J. Zhang, G. Chen, D. Guay, M. Chaker, and D. Ma, Highly active PtAu alloy nanoparticle catalysts for the reduction of 4-nitrophenol, Nanoscale 6, 2125-2130 (2014)

13. B. M. Hunter, J. D. Blakemore, M. Deimund, H. B. Gray, J. R. Winkler, and A. M. Müller, Highly active mixed-metal nanosheet water oxidation catalysts made by pulsed-laser ablation in liquids, J. Am. Chem. Soc. 136 13118-13121 (2014)

14. P. Liu, Y. Liang, X. Lin, C. Wang, and G. Yang, A general strategy to fabricate simple polyoxometalate nanostructures: Electrochemistry-assisted laser ablation in liquid, ACS Nano 5 4748-4755 (2011) 
15. R. Intartaglia, G. Das, K. Bagga, A. Gopalakrishnan, A. Genovese, M. Povia, E. Di Fabrizio, R. Cingolani, A. Diaspro, and F. Brandi, Laser synthesis of ligand-free bimetallic nanoparticles for plasmonic applications, Phys. Chem. Chem. Phys. 15, 3075-3082 (2013)

16. A. Neumeister, J. Jakobi, C. Rehbock, J. Moysig, and S. Barcikowski, Monophasic ligandfree alloy nanoparticle synthesis determinants during pulsed laser ablation of bulk alloy and consolidated microparticles in water, Phys. Chem. Chem. Phys. 16, 23671-23678 (2014)

17. R. M. Tilaki, A. Iraji Zad, S. M. Mahdavi, Stability, size and optical properties of silver nanoparticles prepared by laser ablation in different carrier media, App. Phys. A 84, 215219 (2006)

18. N. Bärsch, J. Jakobi, S. Weiler, and S. Barcikowski, Pure colloidal metal and ceramic nanoparticles from high-power picosecond laser ablation in water and acetone, Nanotechnology 20, 445603 (2009)

19. B. Gökce, D. D. VantZand, A Menendez-Manjon, and S. Barcikowski, Ripening kinetic of laser-generated plasmonic nanoparticle in different solvents, Chem. Phys. Lett. 626, 96-101 (2015)

20. A. Menendez-Manjon, B. Chichkov, and S. Barcikowski, Influence of water temperature on the hydrodynamic diameter of gold nanoparticles from laser ablation, J. Phys. Chem. C 114, 2499-2504 (2010)

21. F. Mafuné, J.-y. Kohno, Y. Takeda, T. Kondow, and H. Sawabe, Formation of gold nanoparticles by laser ablation in aqueous solution of surfactant, J. Phys. Chem. B 105, 5114-5120 (2001)

22. G. Compagnini, A. A. Scalisi, O. Puglisi, and C. Spinella, Synthesis of gold colloids by laser ablation in thiol-alkane solutions, J. Mater. Res. 19, 2795-2798 (2004)

23. J. P. Sylvestre, A. V. Kabashin, E. Sacher, M. Meunier, and J. H. T. Luong, Stabilization and size control of gold nanoparticles during laser ablation in aqueouscyclodextrins, J. Am. Chem. Soc. 126, 7176-7177 (2004)

24. T. Tsuji, D-H. Thang, Y. Okazaki, M. Nakanishi, Y. Tsuboi, and M. Tsuji, Preparation of silver nanoparticle by laser ablation in polyvinylpyrrolidone solutions, Appl. Surf. Sci. 254, 5224-5230 (2008)

25. C. Rehbock, V. Merk, L. Gamrad, R. Streubel, and S. Barcikowski, Size control of laserfabricated surfactant-free gold nanoparticles with highly diluted electrolytes and their subsequent bioconjugation, Phys. Chem. Chem. Phys. 15, 3057-3067 (2013)

26. V. Amendola and M. Meneghetti, Controlled size manipulation of free goldnanoparticles by laser irradiation and their facile bioconjugation, J. Mater. Chem. 17, 4705-4710 (2007)

27. S. Hashimoto, D. Werner, and T. Uwada, Studies on the interaction of pulsed lasers with plasmonic gold nanoparticles toward light manipulation, heat management, and nanofabrication, J. Photochem. Photobiol. C: Photochem. Rev. 13, 28-54 (2012)

28. S. Besner, A. V. Kabashin, F. M. Winnik, and M. Meunier, Synthesis of size-tunable polymer-protected gold nanoparticle by femtosecond laser-based ablation and seed growth, J. Phys. Chem. C 113, 9526-9531 (2009) 
29. A. Pyatenko, H. Q. Wang, N. Koshizaki, and T. Tsuji, Mechanism of pulse laser interaction with colloidal nanoparticles, Laser Photonics Rev. 7, 596-604 (2013)

30. H. Wang, A. Pyatenko, K. Kawaguchi, X. Li, Z. Swiatkowska-Warkocka, and N.Koshizaki, Selective pulsed heating for the synthesis of semiconductor and metal submicrometer spheres, Angew. Chem. Int. Ed. 49, 6361-6364 (2010)

31. Z. Yan, G. Compagnini, and D. B. Chrisey, Generation of AgCl cubes by excimer ablation of bulk $\mathrm{Ag}$ in aqueous $\mathrm{NaCl}$ solutions, J. Phys. Chem. C 115, 5058-5062 (2011)

32. Z. Yan, R. Bao, Y. Huang, and D. B. Chrisey, Hollow particles formed on laser-induced bubbles by excimer laser ablation in liquid, J. Phys. Chem. C 114, 11370-11374 (2010)

33. R. N. W. Zijie Yan, Ruqiang Bao, and D. B. Chrisey, Hollow nanoparticle generation on laser-induced cavitation bubbles via bubble interface pinning, Appl. Phys. Lett. 97, 124106 (2011)

34. D. Zhang, B. Gökce, C. Notthoff, and S. Barcikowski, Layered Seed-Growth of AgGe football-like mircospheres via precursor-free picosecond laser synthesis in water, Sci. Rep. 5, $13661(2015)$

35. S. R. J. Pearce, S. J. Henley, F. Claeyssens, P. W. May, K. R. Hallam, J. A. Smith, and K. N. Rosser, Production of nanocrystalline diamond by laser ablation at the solid/liquid interface, Diamond Relat. Mater. 13, 661-665 (2004)

36. G. W. Yang, Laser ablation in liquids: Applications in the synthesis of nanocrystals, Prog. Mater. Sci. 52, 648-698 (2007)

37. V. Amendola and M. Meneghetti, Laser ablation synthesis in solution and size manipulation of noble metal nanoparticles, Phys. Chem. Chem. Phys. 11, 3805-3821 (2009)

38. N. V. Tarasenko and A. V. Butsen, Laser synthesis and modification of composite nanoparticles in liquids, Quantum Electron. 40, 986-1003 (2010)

39. T. Asahi, F. Mafune, C. Rehbock, and S. Barcikowski, Strategies to harvest the unique properties of laser-generated nanomaterials in biomedical and energy applications, Appl. Surf. Sci. 348, 1-3 (2015)

40. C. Rehbock, J. Jakobi, L. Gamrad, S. van der Meer, D. Tiedemann, U. Taylor, W. Kues, D. Rath, and S. Barcikowski, Current state of laser synthesis of metal and alloy nanoparticles as ligand-free reference materials for nano-toxicological assays, Beilstein J. Nanotechnol. 5, 1523-1541 (2014)

41. V. Amendola and M. Meneghetti, What controls the composition and the structure of nanomaterials generated by laser ablation in liquid solution? Phys. Chem. Chem. Phys. 15, 3027-3046 (2013)

42. A. Tamura, T. Sakka, K. Fukami, and Y. H. Ogata, Dynamics of cavitation bubbles generated by multi-pulse laser irradiation of a solid target in water, Appl. Phys. A 112, 209$213(2013)$ 
43. S. Ibrahimkutty, P. Wagener, A. Menzel, A. Plech, and S. Barcikowski, Nanoparticle formation in a cavitation bubble after pulsed laser ablation in liquid studied with high time resolution small angle x-ray scattering, Appl. Phys. Lett. 101, 103104 (2012)

44. P. Wagener, S. Ibrahimkutty, A. Menzel, A. Plech, and S. Barcikowski, Dynamics of silver nanoparticle formation and agglomeration inside the cavitation bubble after pulsed laser ablation in liquid, Phys. Chem. Chem. Phys. 15, 3068-3074 (2013)

45. S. Ibrahimkutty, P. Wagener, T. dos Santos Rolo, D. Karpov, A. Menzel, T. Baumbach, S. Barcikowski, and A. Plech, A hierarchical view on material formation during pulsed-laser synthesis of nanoparticles in liquid, Sci. Rep. 5, 16313 (2015)

46. J. Lam, J. Lombard, C. Dujardin, G. Ledoux, S. Merabia, and D. Amans, Dynamical study of bubble expansion following laser ablation in liquid, Appl. Phys. Lett. 108, 074104 (2016)

47. J. Lam, D. Amans, F. Chaput, M. Diouf, G. Ledoux, N. Mary, K. Masenelli-Varlot, V. Motto-Ros, and C. Dujardin, $\gamma-\mathrm{Al}_{2} \mathrm{O}_{3}$ nanoparticles synthesised by pulsed laser ablation in liquids: a plasma analysis, Phys. Chem. Chem. Phys. 16, 963-973 (2014)

48. M. Dell'Aglio, R. Gaudiuso, O. De Pascale, and A. De Giacomo, Mechanisms and processes of pulsed laser ablation in liquids during nanoparticle production, Appl. Surf. Sci. 348, 4-9 (2015)

49. E. Giorgetti, P. Marsili, M. Muniz-Miranda, C. Gellini, and F. Giammanco, Spectroscopic evidence of positive clusters in Ag colloids obtained by laser ablation in aqueous solutions, Appl. Phys. A 117, 327-331 (2014)

50. S. Jendrzej, B. Gökce, V. Amendola, and S. Barcikowski, Barrierless growth of precursorfree, ultrafast laser-fragmented noble metal nanoparticles by colloidal atom clusters - A kinetic in situ study, J. Colloid Interface Sci. 463, 299-307 (2016)

51. T. E. Itina, On nanoparticle formation by laser ablation in liquids, J. Phys. Chem. C 115, 5044-5048 (2011)

52. M. E. Povarnitsyn and T. E. Itina, Hydrodynamic modeling of femtosecond laser ablation of metals in vacuum and in liquid, Appl. Phys. A 117, 175-178 (2014)

53. M. E. Povarnitsyn, T. E. Itina, P. R. Levashov, and K. V. Khishchenko, Mechanisms of nanoparticle formation by ultra-short laser ablation of metals in liquid environment, Phys. Chem. Chem. Phys. 15, 3108 (2013)

54. M. P. Allen and D. J. Tildesley, Computer Simulation of Liquids (Clarendon Press: Oxford, 1990, 1987)

55. D. Frenkel and B. Smit, Understanding Molecular Simulation: From Algorithms to Applications (Academic Press: San Diego, 1996)

56. L. V. Zhigilei, E. Leveugle, B. J. Garrison, Y. G. Yingling, and M. I. Zeifman, Computer simulations of laser ablation of molecular substrates, Chem. Rev. 103, 321-348 (2003)

57. L. V. Zhigilei, Z. Lin, D. S. Ivanov, E. Leveugle, W. H. Duff, D. Thomas, C. Sevilla, and S. J. Guy, Atomic/molecular-level simulations of laser-materials interactions, in LaserSurface Interactions for New Materials Production: Tailoring Structure and Properties, 
Springer Series in Materials Science, Vol. 130, Edited by A. Miotello and P. M. Ossi (Springer Verlag: New York, 2010), pp. 43-79.

58. C. Wu, E. T. Karim, A. N. Volkov, and L. V. Zhigilei, Atomic movies of laser-induced structural and phase transformations from molecular dynamics simulations, in Lasers in Materials Science, Springer Series in Materials Science, Vol. 191, Edited by M. Castillejo, P. M. Ossi, and L. V. Zhigilei (Springer International Publishing Switzerland, 2014), pp. 67-100.

59. E. T. Karim, C. Wu, and L. V. Zhigilei, Molecular dynamics simulations of laser-materials interactions: General and material-specific mechanisms of material removal and generation of crystal defects, in Fundamentals of Laser-Assisted Micro- and Nanotechnologies, Springer Series in Materials Science, Vol. 195, Edited by V. P. Veiko and V. I. Konov (Springer International Publishing Switzerland, 2014), pp. 27-49.

60. D. M. Bubb, S. M. O’Malley, J. Schoeffling, R. Jimenez, B. Zinderman, and S. Yi, Size control of gold nanoparticles produced by laser ablation of thin films in an aqueous environment, Chem. Phys. Lett. 565, 65-68 (2013)

61. S. Scaramuzza, M. Zerbetto, and V. Amendola, Synthesis of gold nanoparticles in liquid environment by laser ablation with geometrically confined configurations: Insights to improve size control and productivity, J. Phys. Chem. C 120, 9453 (2016)

62. D. S. Ivanov and L. V. Zhigilei, Combined atomistic-continuum modeling of short pulse laser melting and disintegration of metal films, Phys. Rev. B 68, 064114 (2003)

63. L. V. Zhigilei, Z. Lin, and D. S. Ivanov, Atomistic modeling of short pulse laser ablation of metals: Connections between melting, spallation, and phase explosion, J. Phys. Chem. C 113, 11892-11906 (2009)

64. C. Wu and L. V. Zhigilei, Microscopic mechanisms of laser spallation and ablation of metal targets from large-scale molecular dynamics simulations, Appl. Phys. A 114, 11-32 (2014)

65. C. Wu, M. S. Christensen, J.-M. Savolainen, P. Balling, and L. V. Zhigilei, Generation of sub-surface voids and a nanocrystalline surface layer in femtosecond laser irradiation of a single crystal Ag target, Phys. Rev. B 91, 035413 (2015)

66. S. I. Anisimov, B. L. Kapeliovich, and T. L. Perel'man, Electron emission from metal surfaces exposed to ultrashort laser pulses, Sov. Phys. JETP 39, 375-377 (1974)

67. S. M. Foiles, M. I. Baskes, and M. S. Daw, Embedded-atom-method functions for the fcc metals Cu, Ag, Au, Ni, Pd, Pt, and their alloys, Phys. Rev. B 33, 7983-7991 (1986)

68. A. F. Voter and S. P. Chen, Accurate interatomic potentials for Ni, Al, and $\mathrm{Ni}_{3} \mathrm{Al}$, Mat. Res. Soc. Symp. Proc. 82, 175-180 (1999)

69. S. M. Foiles and J. B. Adams, Thermodynamic properties of fcc transition metals as calculated with embedded-atom method, Phys. Rev. B 40, 5909-5915 (1989)

70. C. Wu, D. A. Thomas, Z. Lin, and L. V. Zhigilei, Runaway lattice-mismatched interface in an atomistic simulation of femtosecond laser irradiation of $\mathrm{Ag}$ film - $\mathrm{Cu}$ substrate system, Appl. Phys. A 104, 781-792 (2011) 
71. D. R. Lide (ed.), CRC Handbook of Chemistry and Physics, $79^{\text {th }}$ edn. (CRC Press: Boca Raton, FL, 1999)

72. E. T. Karim, M. Shugaev, C. Wu, Z. Lin, R. F. Hainsey, and L. V. Zhigilei, Atomistic simulation study of short pulse laser interactions with a metal target under conditions of spatial confinement by a transparent overlayer, J. Appl. Phys. 115, 183501 (2014)

73. B. J. Garrison, T. E. Itina, and L. V. Zhigilei, The limit of overheating and the threshold behavior in laser ablation, Phys. Rev. E 68, 041501 (2003)

74. A. Miotello and R. Kelly, Laser-induced phase explosion: new physical problems when a condensed phase approaches the thermodynamic critical temperature, Appl. Phys. A 69, S67-S73 (1999)

75. N. M. Bulgakova and A. V. Bulgakov, Pulsed laser ablation of solids: transition from normal vaporization to phase explosion, Appl. Phys. A 73, 199-208 (2001)

76. R. W. Ohse and H. von Tippelskirch, The critical constants of the elements and of some refractory materials with high critical temperatures. (A review), High Temp.-High Press. 9, 367-385 (1977)

77. Z. Lin, L. V. Zhigilei, and V. Celli, Electron-phonon coupling and electron heat capacity of metals under conditions of strong electron-phonon nonequilibrium, Phys. Rev. B 77, 075133 (2008)

78. R. H. M. Groeneveld, R. Sprik, and A. Lagendijk, Femtosecond spectroscopy of electronelectron and electron-phonon energy relaxation in Ag and Au, Phys. Rev. B 51, 1143311445 (1995)

79. K. C. Mills, B. J. Monaghan, and B. J. Keene, Thermal conductivities of molten metals: Part 1 Pure metals, Int. Mater. Rev. 41, 209-242 (1996)

80. H. J. Kunze, Introduction to Plasma Spectroscopy (Springer, Berlin, 2009), pp. 242.

81. H. R. Griem, High-density corrections in plasma spectroscopy, Phys. Rev. 128, 997-1003 (1962)

82. F. Taccogna, Nucleation and growth of nanoparticles in a plasma by laser ablation in liquid, J. Plasma Phys. 81, 495810509 (2015)

83. L. V. Zhigilei, P. B. S. Kodali, and B. J. Garrison, Molecular dynamics model for laser ablation of organic solids, J. Phys. Chem. B 101, 2028-2037 (1997)

84. D. J. Phares and A. R. Srinivasa, Molecular dynamics with molecular temperature, J. Phys. Chem. A 108, 6100-6108 (2004)

85. W. M. Jacobs, D. A. Nicholson, H. Zemer, A. N. Volkov, and L. V. Zhigilei, Acoustic energy dissipation and thermalization in carbon nanotubes: Atomistic modeling and mesoscopic description, Phys. Rev. B 86, 165414 (2012)

86. M. Tabetah, A. Matei, C. Constantinescu, N. P. Mortensen, M. Dinescu, J. Schou, and L. V. Zhigilei, The minimum amount of "matrix" needed for matrix-assisted pulsed laser deposition of biomolecules, J. Phys. Chem. B 118, 13290-13299 (2014) 
87. H. Okumura and F. Yonezawa, Reliable determination of the liquid-vapor critical point by the NVT plus test particle method, J. Phys. Soc. Jpn. 70, 1990-1994 (2001)

88. A. Michaelides, V. A. Ranea, P. L. deAndres, and D.A. King, General model for water monomer adsorption on close-packed transition and noble metal surfaces, Phys. Rev. Lett 90, $216102(2003)$

89. J. Ren and S. Meng, First-principles study of water on copper and noble metal (110) surfaces, Phys. Rev. B 77, 054110 (2008)

90. Z. E. Hughes, L. B. Wright, and T. R. Walsh, Biomolecular adsorption at aqueous silver interfaces: First-principles calculations, polarizable force-field simulations, and comparisons with gold, Langmuir 29, 13217-13229 (2013)

91. A. Michaelides and D.A. King, Insight into $\mathrm{H}_{2} \mathrm{O}$-ice adsorption and dissociation on metal surfaces from first-principles simulations, Phys. Rev. B 69, 113404 (2004)

92. Y. G. Yingling, L. V. Zhigilei, B. J. Garrison, Photochemical fragmentation processes in laser ablation of organic solids, Nucl. Instr. Meth. B 180, 171-175 (2001)

93. Y. G. Yingling, L. V. Zhigilei, B. J. Garrison, The role of photochemical fragmentation in laser ablation: a molecular dynamics study, J. Photochem. Photobiol. A 145, 173-181 (2001)

94. J. Lam, D. Amans, C. Dujardin, G. Ledoux, and A.-R. Allouche, Atomistic mechanisms for the nucleation of aluminum oxide nanoparticles, J. Phys. Chem. A 119, 8944-8949 (2015)

95. L. V. Zhigilei and B. J. Garrison, Pressure waves in microscopic simulations of laser ablation, Mat. Res. Soc. Symp. Proc. 538, 491-496 (1999)

96. C. Schäfer, H. M. Urbassek, L. V. Zhigilei, and B. J. Garrison, Pressure-transmitting boundary conditions for molecular dynamics simulations, Comp. Mater. Sci. 24, 421-429 (2002)

97. D. Bäuerle, Laser Processing and Chemistry (Springer-Verlag, Berlin Heidelberg, 2000)

98. S.-S. Wellershoff, J. Hohlfeld, J. Güdde, E. Matthias, The role of electron-phonon coupling in femtosecond laser damage of metals, Appl. Phys. A 69, S99-S107 (1999)

99. C. M. Rouleau, C.-Y. Shih, C. Wu, L. V. Zhigilei, A. A. Puretzky, and D. B. Geohegan, Nanoparticle generation and transport resulting from femtosecond laser ablation of ultrathin metal films: Time-resolved measurements and molecular dynamics simulations, Appl. Phys. Lett. 104, 193106 (2014)

100. J. M. J. Santillán, M. B. Fernández van Raap, P. M. Zélis, D. Coral, D. Muraca, D. C. Schinca, L. B. Scaffardi, Ag nanoparticles formed by femtosecond pulse laser ablation in water: self-assembled fractal structures, J. Nanopart. Res. 17, 86 (2015)

101. A. Vrij, Possible mechanism for the spontaneous rupture of thin, free liquid films, Discuss. Faraday Soc. 42, 23-33 (1966)

102. H. J. Kull, Theory of the Rayleigh-Taylor instability, Phys. Rep. 206, 197-325 (1991) 
103. K. Kadau, T. C. Germann, N. G. Hadjiconstantinou, P. S. Lomdahl, G. Dimonte, B. L. Holian, and B. J. Alder, Nanohydrodynamics simulations: An atomistic view of the Rayleigh-Taylor instability, Proc. Natl. Acad. Sci. USA 101, 5851-5855 (2004)

104. L. V. Zhigilei and B. J. Garrison, Microscopic mechanisms of laser ablation of organic solids in the thermal and stress confinement irradiation regimes, J. Appl. Phys. 88, 12811298 (2000) 
Tables

\begin{tabular}{|c|c|c|c|}
\hline properties of water & experiment & CG model & $\Delta, \%$ \\
\hline density, $\rho, \mathrm{g} / \mathrm{cm}^{3}$ & 1.0 & 1.0 & 0 \\
\hline heat capacity, $c_{p}, \mathrm{~J} /(\mathrm{kg} \mathrm{K})$ & $4.2 \times 10^{3}$ & $4.2 \times 10^{3}$ & 0 \\
\hline bulk modulus, $K, \mathrm{GPa}$ & 2.2 & 1.8 & 18 \\
\hline speed of sound, $c_{s}, \mathrm{~m} / \mathrm{s}$ & 1483 & 1342 & 9 \\
\hline melting temperature, $T_{m}, \mathrm{~K}$ & 273 & 330 & 21 \\
\hline critical temperature, $T_{c}, \mathrm{~K}$ & 647 & 520 & 20 \\
\hline critical density, $\rho_{c}, \mathrm{~g} / \mathrm{cm}^{3}$ & 0.322 & 0.398 & 24 \\
\hline thermal conductivity, $k, \mathrm{~W} /(\mathrm{m} \mathrm{K})$ & 0.6 & 0.13 & 78 \\
\hline viscosity, $\eta, \mathrm{cP}$ & 0.894 & 0.910 & 2 \\
\hline surface energy, $\gamma, \mathrm{J} / \mathrm{m}^{2}$ & 0.072 & 0.073 & 1 \\
\hline
\end{tabular}

Table 1. Properties of the coarse-grained model parametrized for water and the corresponding experimental values. The values of $\rho, c_{p}, K, c_{s}, k, \eta$, and $\gamma$ are listed for a temperature of $300 \mathrm{~K}$. The experimental values are from Ref. [71]. 


\section{Figures and figure captions}

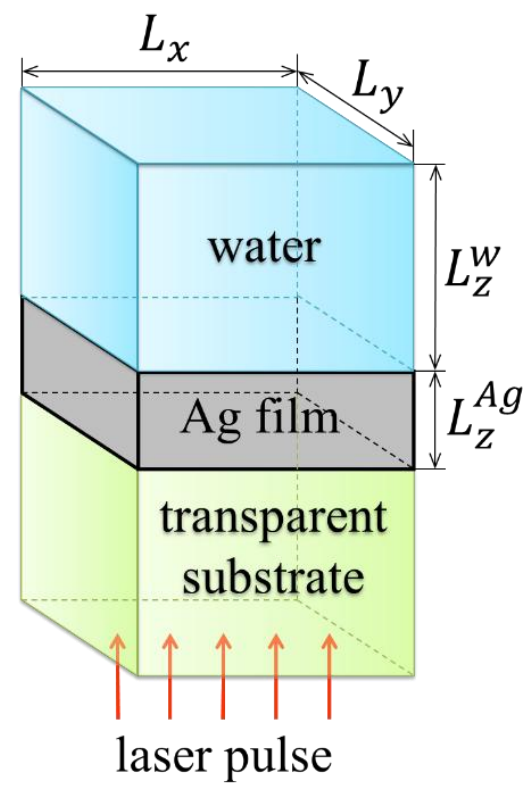

Figure 1. Schematic representation of the computational setup for simulation of short pulse laser interaction with a thin silver film in water deposited on a transparent silica substrate. The grey rectangular parallelepiped shows the initial location of the silver film represented by the TTM-MD model. The blue rectangular parallelepiped shows the initial location of the water represented by the coarse-grained MD model. The initial dimensions of the TTM-MD and coarse-grained MD parts of the system are $L_{x}=L_{y}=100 \mathrm{~nm}, L_{z}^{A g}=20 \mathrm{~nm}$, and $L_{z}^{w}=300 \mathrm{~nm}$. The elastic response of the substrate and the non-reflecting propagation of the laser-induced pressure wave in water environment are represented by acoustic impedance matching boundary conditions applied at the substrate-film interface and at the top of the coarse-grained MD part of the system. The laser pulse is directed from the bottom of the figure, through the transparent substrate. Periodic boundary conditions are applied in the lateral directions, perpendicular to the surface normal of the film. 


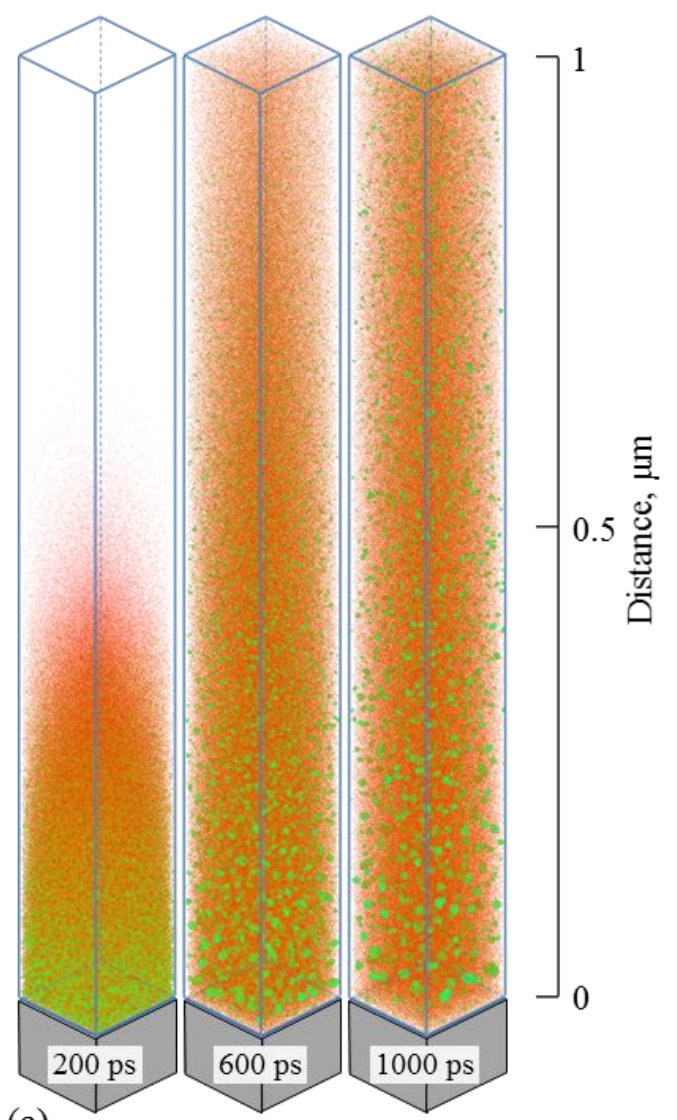

(a)

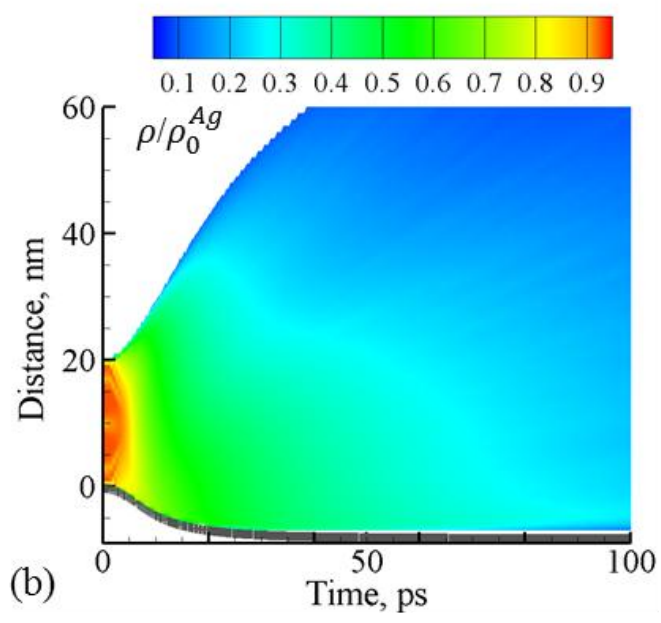

Figure 2. Snapshots of atomic configurations (a) and density contour plot (b) obtained in a MD simulation of laser ablation of a $20 \mathrm{~nm} \mathrm{Ag}$ film in vacuum irradiated by a $40 \mathrm{fs}$ laser pulse at an absorbed fluence of $360 \mathrm{~J} / \mathrm{m}^{2}$. The transparent silica substrate is represented in (a) by the grey rectangular parallelepiped. The Ag atoms are colored by their potential energies, so that the green atoms belong to liquid droplets and the red ones are the vapor-phase atoms. The density in (b) is normalized by the initial density of solid silver at $300 \mathrm{~K}, \rho_{0}^{A g}$. 

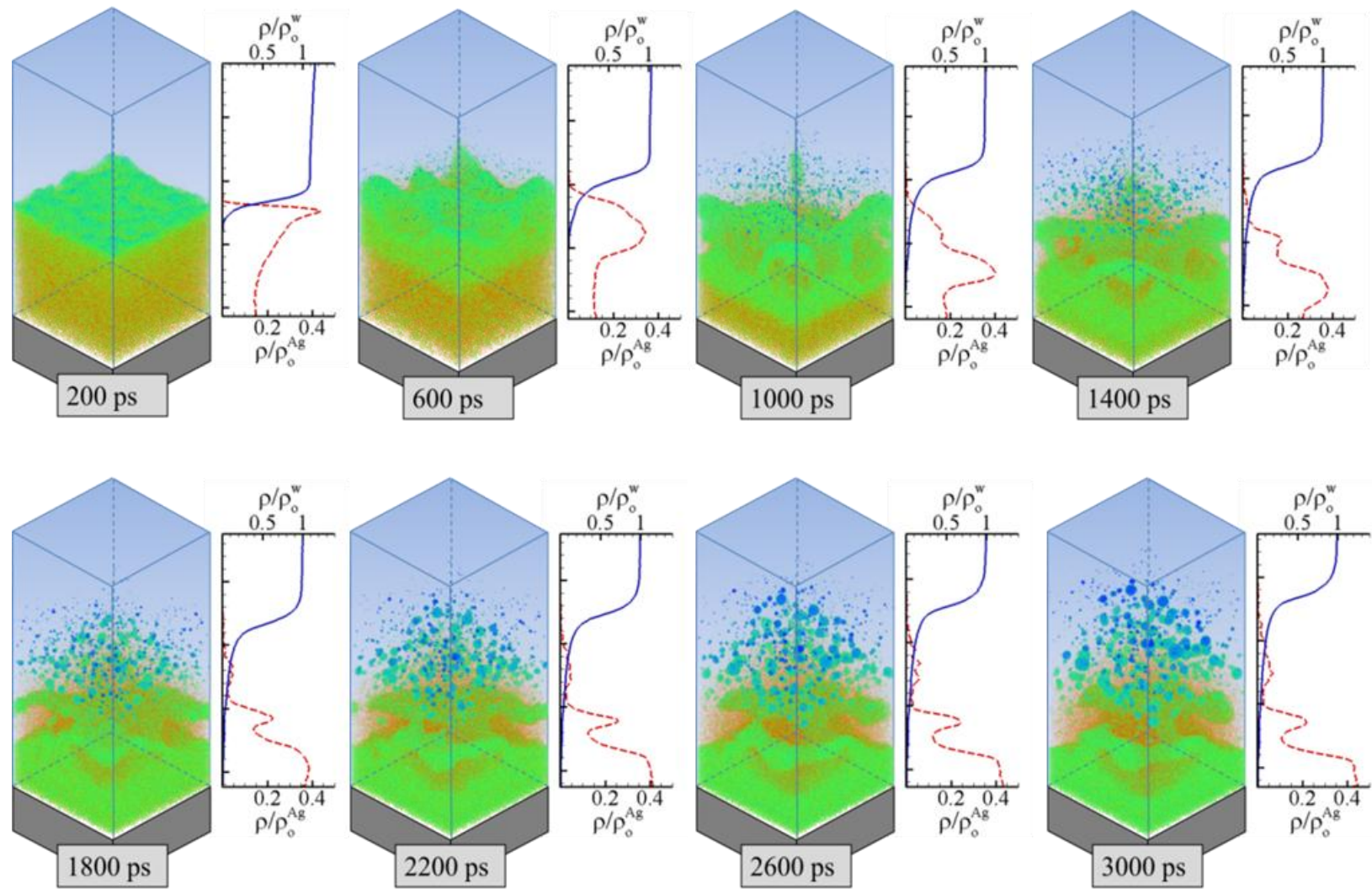

Figure 3. Snapshots of atomic configurations obtained in a MD simulation of laser ablation of a $20 \mathrm{~nm} \mathrm{Ag}$ film in water irradiated by a $40 \mathrm{fs}$ laser pulse at an absorbed fluence of $400 \mathrm{~J} / \mathrm{m}^{2}$. The transparent silica substrate is represented by the grey rectangular parallelepiped. A 200-nm-wide region above the substrate is shown in the snapshots. The Ag atoms are colored by their potential energies, in the range from -2.5 to $-1.5 \mathrm{eV}$, so that the blue atoms belong to solidified nanoparticles and the red ones are the vapor-phase atoms. The water molecules are blanked to expose the nanoparticle formation, and the presence of water is shown schematically by the blue background. The degree of water-silver mixing is illustrated by density plots shown as functions of distance from the substrate for both water and silver on the right sides of the snapshots. The values of the water and silver densities in these plots are normalized by the initial densities of liquid water and solid silver at $300 \mathrm{~K}, \boldsymbol{\rho}_{\mathbf{0}}^{\boldsymbol{w}}$ and $\boldsymbol{\rho}_{\mathbf{0}}^{\boldsymbol{A} \boldsymbol{g}}$, respectively. 

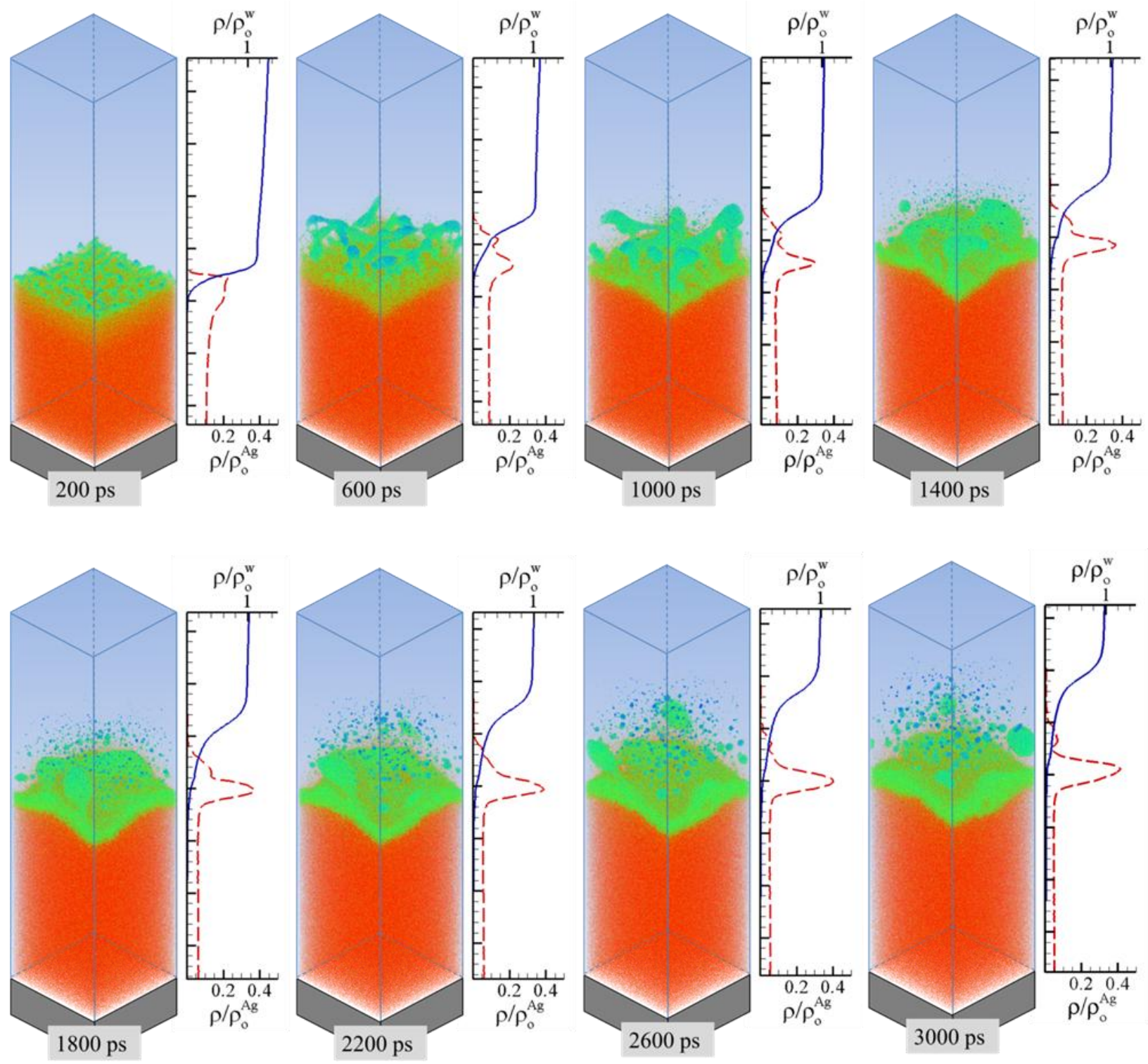

Figure 4. Snapshots of atomic configurations obtained in a MD simulation of laser ablation of a $20 \mathrm{~nm} \mathrm{Ag}$ film in water irradiated by a $40 \mathrm{fs}$ laser pulse at an absorbed fluence of $700 \mathrm{~J} / \mathrm{m}^{2}$. The transparent silica substrate is represented by the grey rectangular parallelepiped. A 350-nm-wide region above the substrate is shown in the snapshots. The Ag atoms are colored by their potential energies, in the range from -2.5 to $-1.5 \mathrm{eV}$, so that the blue atoms belong to solidified nanoparticles and the red ones are the vapor-phase atoms. The water molecules are blanked to expose the nanoparticle formation, and the presence of water is shown schematically by the blue background. The degree of water-silver mixing is illustrated by density plots shown as functions of distance from the substrate for both water and silver on the right sides of the snapshots. The values of the water and silver densities in these plots are normalized by the initial densities of liquid water and solid silver at $300 \mathrm{~K}, \boldsymbol{\rho}_{\mathbf{0}}^{\boldsymbol{w}}$ and $\boldsymbol{\rho}_{\mathbf{0}}^{\boldsymbol{A}}$, respectively. 

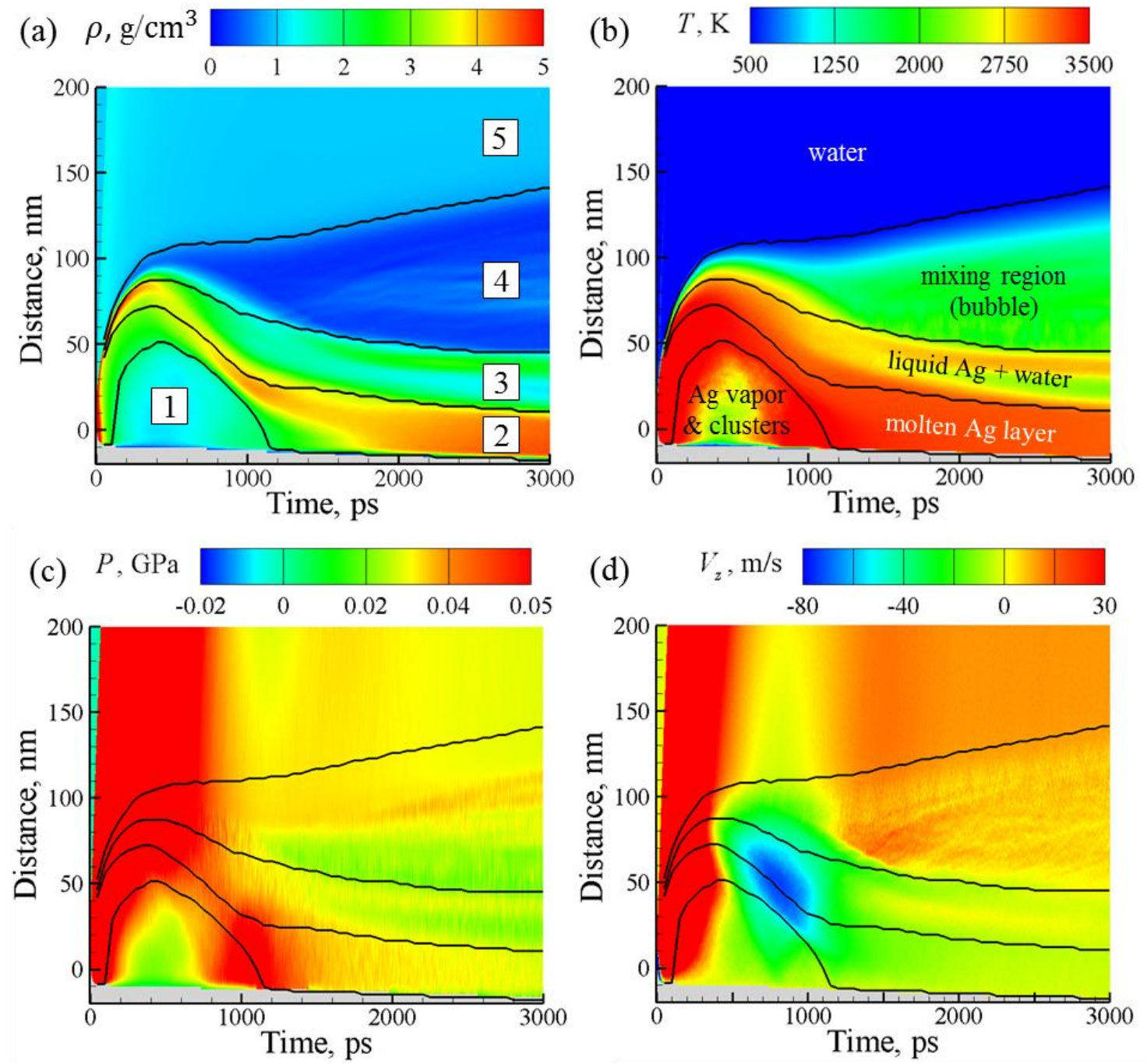

Figure 5. The contour plots of the initial spatial and time evolution of the density (a), lattice temperature (b), pressure (c), and collective velocity in the direction away from the substrate (d) predicted in a MD simulation of laser ablation of a $20 \mathrm{~nm} \mathrm{Ag}$ film in water irradiated by a $40 \mathrm{fs}$ laser pulse at an absorbed fluence of $400 \mathrm{~J} / \mathrm{m}^{2}$. The snapshots from the simulation are shown in Fig. 3. The laser pulse is directed along the $y$ axis from the bottom of the contour plots, through a silica substrate shown as a grey area. The black lines separate five distinct regions labeled by numbers in (a): Region 1 corresponds the gaseous mixture of Ag vapor and small clusters, region 2 is a compact superheated molten Ag, region 3 is a "terrain" of liquid Ag structures and droplets mixed with water, region 4 is a $\mathrm{Ag}$ - supercritical water mixing region where $\mathrm{Ag}$ nanoparticles form through condensation and growth, and region 5 is pure liquid water. The quantitative criteria used for identification of the regions are explained in the test. 

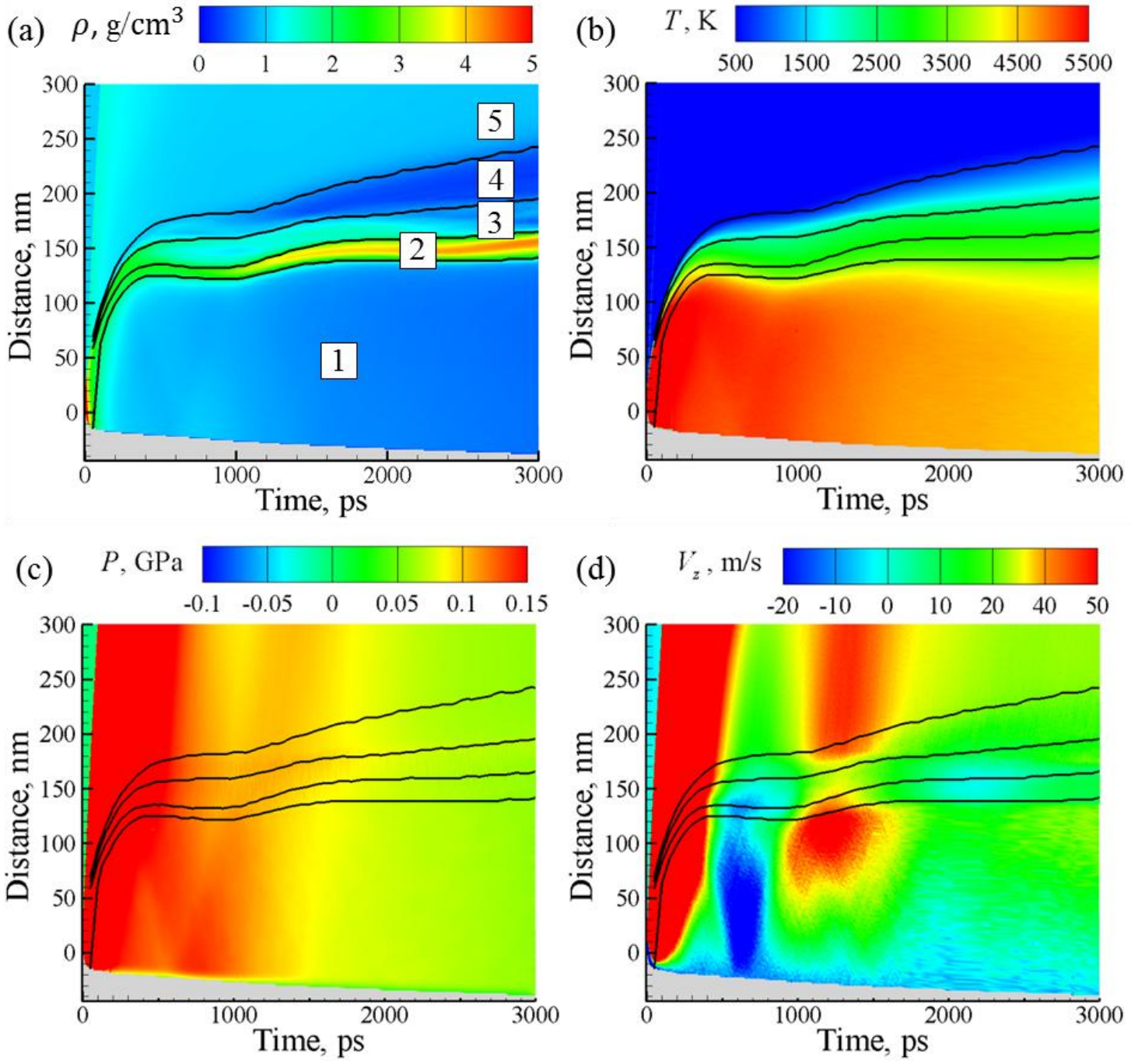

Figure 6. The contour plots of the initial spatial and time evolution of the density (a), lattice temperature (b), pressure (c), and collective velocity in the direction away from the substrate (d) predicted in a MD simulation of laser ablation of a $20 \mathrm{~nm} \mathrm{Ag}$ film in water irradiated by a $40 \mathrm{fs}$ laser pulse at an absorbed fluence of $700 \mathrm{~J} / \mathrm{m}^{2}$. The snapshots from the simulation are shown in Fig. 4. The laser pulse is directed along the $y$ axis from the bottom of the contour plots, through a silica substrate shown as a grey area. The black lines separate five distinct regions labeled by numbers in (a): Region 1 corresponds the gaseous mixture of Ag vapor and small clusters, region 2 is a compact superheated molten Ag, region 3 is a "terrain" of liquid Ag structures and droplets mixed with water, region 4 is a Ag - supercritical water mixing region where Ag nanoparticles form through condensation and growth, and region 5 is pure liquid water. The quantitative criteria used for identification of the regions are explained in the test. 

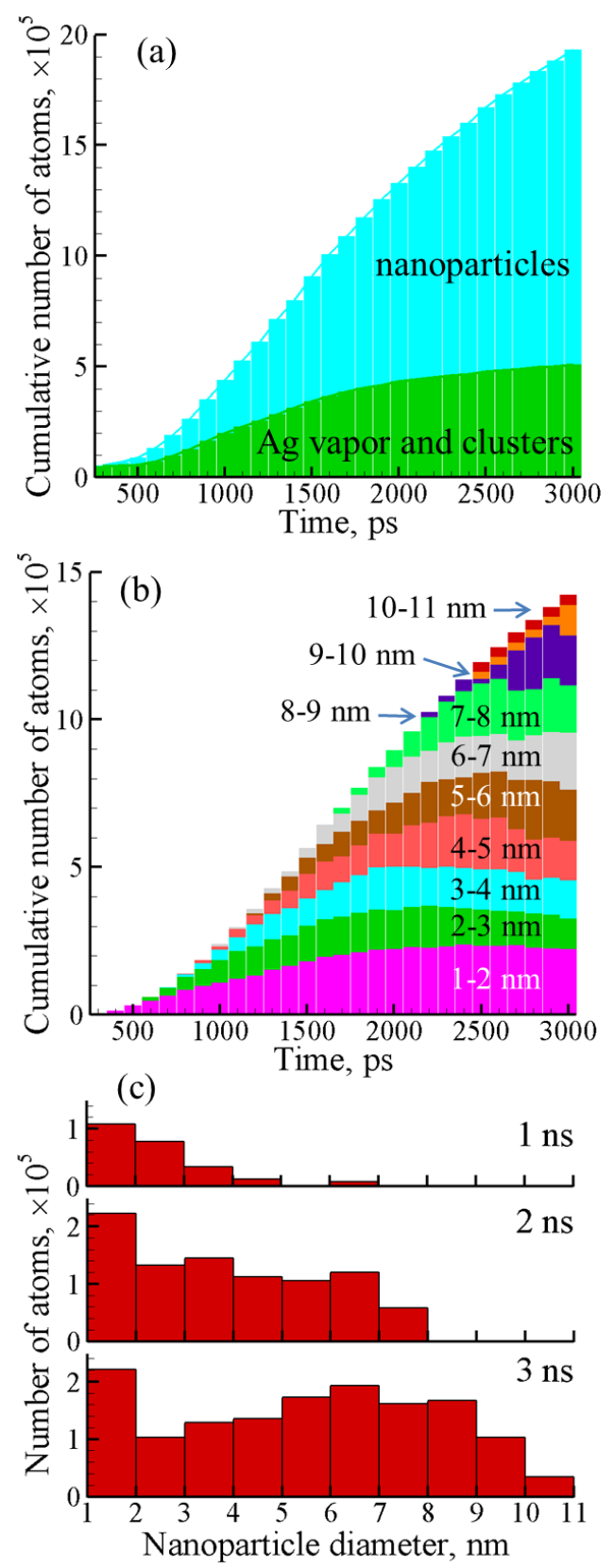

Figure 7. The results of the cluster analysis of the ablation plume generated in a simulation performed at an absorbed fluence of $400 \mathrm{~J} / \mathrm{m}^{2}$. The cumulative number of individual Ag atoms and small clusters with diameters less than $1 \mathrm{~nm}$ (green) and atoms that belong to $\mathrm{Ag}$ nanoparticles with diameters above $1 \mathrm{~nm}$ (blue) are shown in (a). The cumulative numbers of atoms that belong to nanoparticles of different sizes (above $1 \mathrm{~nm}$ ) are shown in (b). The number of atoms in nanoparticles of different sizes (above $1 \mathrm{~nm}$ ) are also shown as histograms for 1, 2 and $3 \mathrm{~ns}$ in (c). The analysis is applied only to the part of the plume above the molten layer, i.e., to the regions 3 and 4 in Fig. 5. 

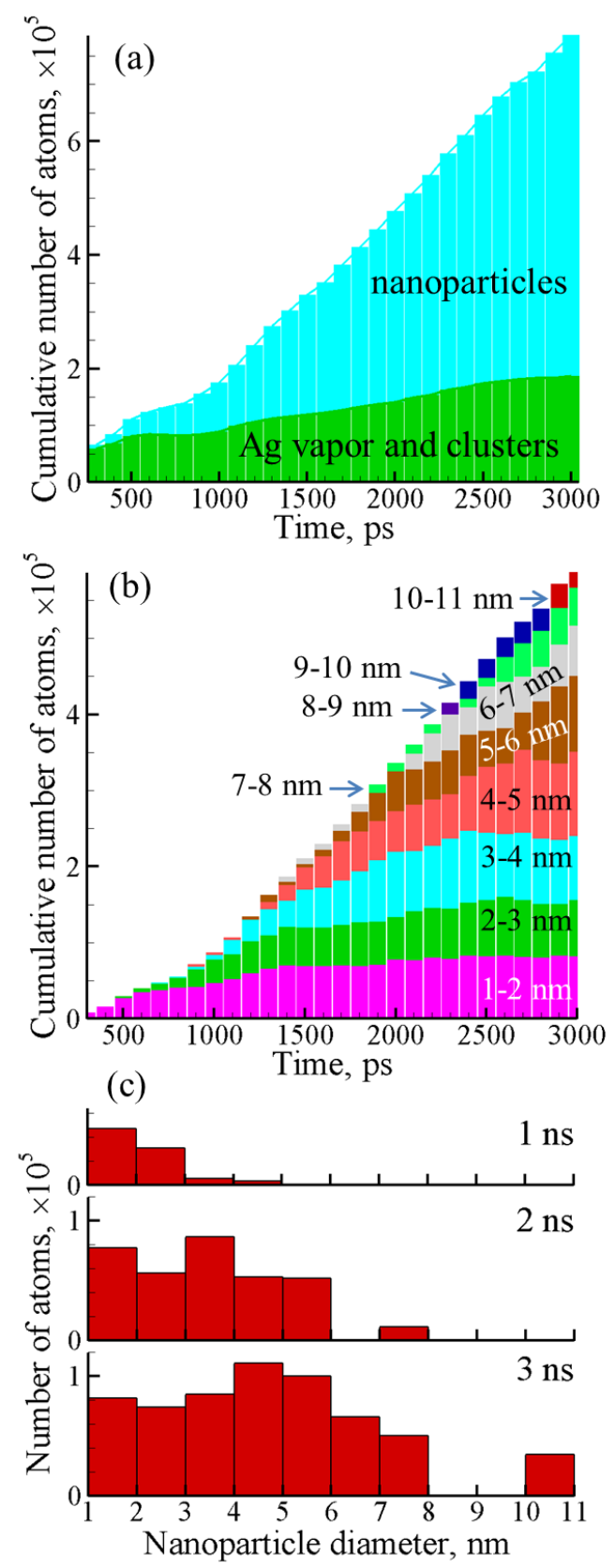

Figure 8. The results of the cluster analysis of the ablation plume generated in a simulation performed at an absorbed fluence of $700 \mathrm{~J} / \mathrm{m}^{2}$. The cumulative number of individual $\mathrm{Ag}$ atoms and small clusters with diameters less than $1 \mathrm{~nm}$ (green) and atoms that belong to $\mathrm{Ag}$ nanoparticles with diameters above $1 \mathrm{~nm}$ (blue) are shown in (a). The cumulative numbers of atoms that belong to nanoparticles of different sizes (above $1 \mathrm{~nm}$ ) are shown in (b). The number of atoms in nanoparticles of different sizes (above $1 \mathrm{~nm}$ ) are also shown as histograms for 1,2 and $3 \mathrm{~ns}$ in (c). The analysis is applied only to the part of the plume above the molten layer, i.e., to the regions 3 and 4 in Fig. 6. 


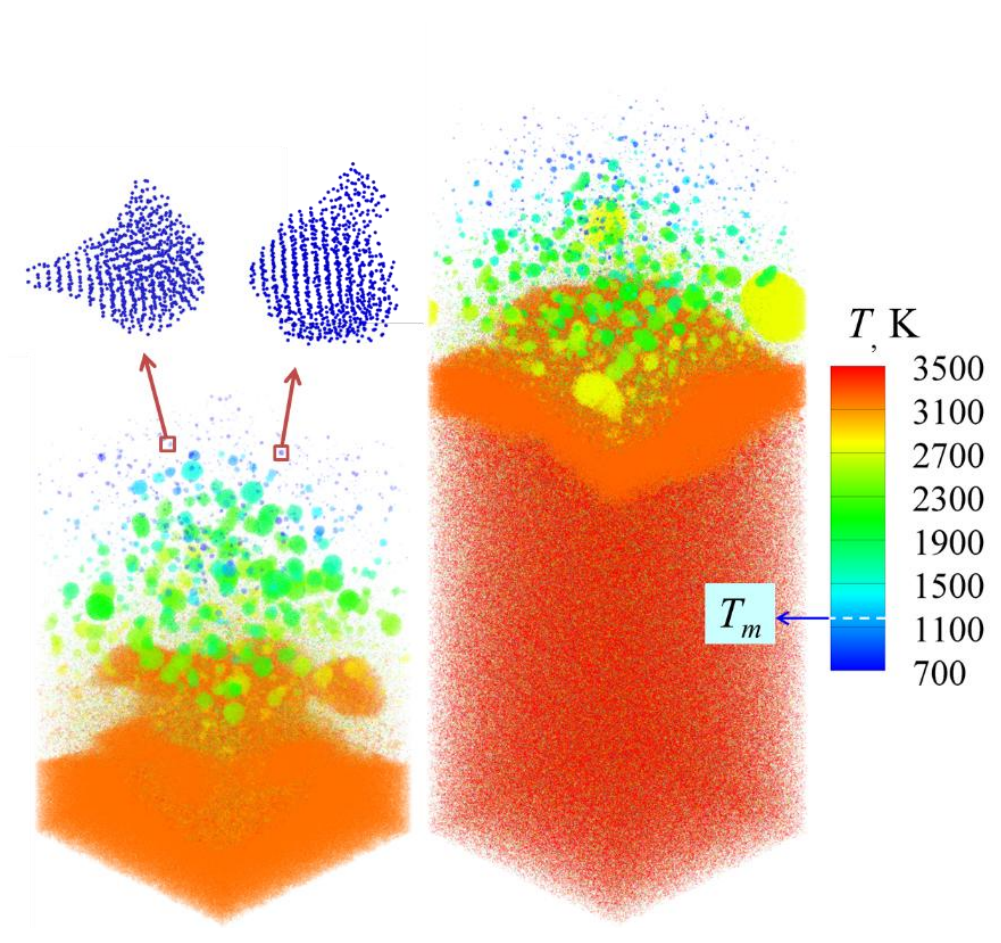

Figure 9. The snapshots of final configurations obtained for $3 \mathrm{~ns}$ after the laser pulse in simulations performed at absorbed fluences of $400 \mathrm{~J} / \mathrm{m}^{2}$ (left) and $700 \mathrm{~J} / \mathrm{m}^{2}$ (right). The atoms in the snapshots are colored by local temperature. The enlarged view of atomic structure of two crystalline nanoparticles located in the upper part of the mixing region (region 4 in Fig. 5) is shown in the left panel. 
Figure for on-line table of content (graphical abstract)
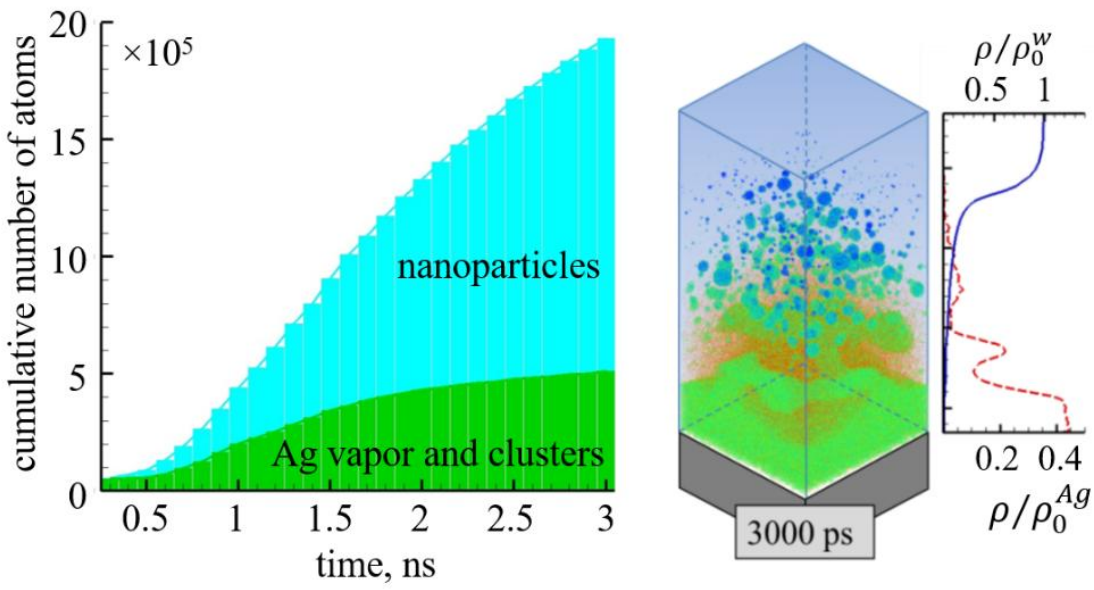